\title{
Quantum mechanics/molecular mechanics minimum free-energy path for accurate reaction energetics in solution and enzymes: Sequential sampling and optimization on the potential of mean force surface
}

\author{
Hao Hu, Zhenyu Lu, Jerry M. Parks, Steven K. Burger, and Weitao Yang ${ }^{\text {a) }}$ \\ Department of Chemistry, Duke University, Durham, North Carolina 27708, USA
}

(Received 3 July 2007; accepted 31 October 2007; published online 16 January 2008)

\begin{abstract}
To accurately determine the reaction path and its energetics for enzymatic and solution-phase reactions, we present a sequential sampling and optimization approach that greatly enhances the efficiency of the $a b$ initio quantum mechanics/molecular mechanics minimum free-energy path (QM/MM-MFEP) method. In the QM/MM-MFEP method, the thermodynamics of a complex reaction system is described by the potential of mean force (PMF) surface of the quantum mechanical $(\mathrm{QM})$ subsystem with a small number of degrees of freedom, somewhat like describing a reaction process in the gas phase. The main computational cost of the QM/MM-MFEP method comes from the statistical sampling of conformations of the molecular mechanical (MM) subsystem required for the calculation of the QM PMF and its gradient. In our new sequential sampling and optimization approach, we aim to reduce the amount of MM sampling while still retaining the accuracy of the results by first carrying out MM phase-space sampling and then optimizing the QM subsystem in the fixed-size ensemble of MM conformations. The resulting QM optimized structures are then used to obtain more accurate sampling of the MM subsystem. This process of sequential MM sampling and QM optimization is iterated until convergence. The use of a fixed-size, finite MM conformational ensemble enables the precise evaluation of the QM potential of mean force and its gradient within the ensemble, thus circumventing the challenges associated with statistical averaging and significantly speeding up the convergence of the optimization process. To further improve the accuracy of the QM/MM-MFEP method, the reaction path potential method developed by Lu and Yang [Z. Lu and W. Yang, J. Chem. Phys. 121, 89 (2004)] is employed to describe the $\mathrm{QM} / \mathrm{MM}$ electrostatic interactions in an approximate yet accurate way with a computational cost that is comparable to classical MM simulations. The new method was successfully applied to two example reaction processes, the classical $\mathrm{S}_{\mathrm{N}} 2$ reaction of $\mathrm{Cl}^{-}+\mathrm{CH}_{3} \mathrm{Cl}$ in solution and the second proton transfer step of the reaction catalyzed by the enzyme 4-oxalocrotonate tautomerase. The activation free energies calculated with this new sequential sampling and optimization approach to the QM/MM-MFEP method agree well with results from other simulation approaches such as the umbrella sampling technique with direct $\mathrm{QM} / \mathrm{MM}$ dynamics sampling, demonstrating the accuracy of the iterative QM/MM-MFEP method. () 2008 American Institute of Physics.
\end{abstract} [DOI: $10.1063 / 1.2816557]$

\section{INTRODUCTION}

Understanding reaction mechanisms and the origins of catalytic proficiency of enzymes are among the most fundamental topics in chemical and biological sciences. Information from experiments is necessary, but often insufficient, to determine the precise mechanism and energetics of a reaction process. Complementary to experiments, computational studies can in principle provide detailed knowledge at the atomic or even the electronic level regarding how a molecular system changes from one state to another. This knowledge includes the reaction path, the structure of the transition state, and the interaction energetics. ${ }^{1}$

In general, computer simulations of chemical reactions face two main challenges: One is the accurate description of the interactions of the molecular system (the potential energy

${ }^{a)}$ Electronic mail: weitao.yang@duke.edu. surface), and the other is sufficient statistical sampling to ensure that the results are converged in a thermodynamic sense. The first challenge usually requires the use of an appropriate level of quantum-mechanical (QM) theory, with $a b$ initio QM being more desirable for the accuracy and consistency of the results. The second challenge can be addressed through extensive phase-space sampling of the system. For small molecule reactions in the gas phase, both challenges can be tackled in a satisfactory manner. With currently available computational resources, the energetics of small molecules can be calculated quantum mechanically. The statistical partition function, required for computing the thermodynamics of the system, can be computed analytically by using a harmonic approximation or sampled directly through numerical simulations.

When a reaction occurs either in solution or in an enzyme, the interactions between the reactive moieties and their surroundings are significant and must therefore be in- 
cluded in the calculations. Consequently, there may be too many degrees of freedom and the harmonic approximation required for calculating the analytic partition function is no longer valid for all relevant degrees of freedom. As a result, direct conformational space sampling becomes necessary even though it is computationally demanding. Normally, the sampling of the complicated multidimensional conformational space is achieved by molecular dynamics (MD) or Monte Carlo (MC) simulations, which can in principle perform effectively for macromolecular systems. In practice, however, the reliability of the results depends critically on the available simulation time.

Because of the excessive computational demands, it is also difficult to describe an entire condensed-phase reaction system quantum mechanically. Instead, the potential energy of a complex reaction system can be described efficiently with a combined quantum mechanical and molecular mechanical (QM/MM) approach. ${ }^{2}$ In this approach, only a portion of the system that makes the most important and direct contributions to the reaction process is described quantum mechanically, while the rest of the system is described by molecular mechanics. The partitioning of the system into two subsystems allows a complex system to be modeled in an accurate, yet affordable manner. With steady improvements in computer speed, the QM/MM approach has been applied to numerous biochemical problems. ${ }^{3-29}$

Even with the savings provided by a reasonably sized QM subsystem in the QM/MM approach, ab initio QM calculations are still quite expensive in general. For the majority of QM/MM applications in the literature, either semiempirical QM methods have been employed to carry out direct conformational sampling of the QM/MM subsystem with $\mathrm{MD}$ or $\mathrm{MC}$, or only a limited number of conformations of the system have been simulated with $a b$ initio QM/MM methods.

When semiempirical QM/MM methods are used, the computational costs are low enough that classical statistical approaches such as umbrella sampling, ${ }^{30}$ potential of mean force (PMF) calculations, and thermodynamic integration can be applied in a straightforward manner. The structural and energetic properties are often obtained by modeling the reaction as a process proceeding along a reaction coordinate or order parameter. Because broad phase-space sampling is attainable, the convergence of the results may be satisfactory. Nonetheless, the results may be less accurate and reliable compared to $a b$ initio QM calculations due to the wellknown deficiencies of semiempirical QM methods.

The limitations of semiempirical QM/MM methods have motivated the development of several $a b$ initio QM/MM methods including the QM-FE method, ${ }^{7,8,31-33}$ the QM(ai)/MM method, ${ }^{4,5,34-38}$ and the QM/MM-FE method. $^{21,24,39,40}$ The QM/MM-FE method developed in our laboratory, and closely related to our development in this report, is comprised of three major ingredients: (1) The pseudobond $a b$ initio QM/MM method, which offers a smooth interface between the QM and MM subsystems and thus provides a well-defined potential energy surface; (2) an efficient, iterative optimization procedure which allows the determination of reaction paths within a realistic enzyme en- vironment; and (3) free-energy perturbation calculations, which provide accurate free energies between QM conformations and take into account the fluctuations of the surrounding enzyme and solvent environment. This method, along with its further extensions, has been successfully applied to the study of many enzymatic reactions and has provided important insights into many biologically relevant reactions. $^{23,28,29,41-46}$ However, the main limitation of the QM/MM-FE method is that the optimization of the reaction path depends on the choice of the initial conformation, ${ }^{47}$ although it is debatable how much this dependence can bias the results in the simulations of enzymatic reactions. ${ }^{48}$ For reactions in solution, however, the dependence of the reaction path on the initial conformation renders the application of the QM/MM-FE method nearly impossible because of the disorder and rapid changes in the positions of the solvent molecules.

To eliminate the conformational dependence of the MM environment on the reaction path, we recently developed the QM/MM minimum free-energy path (QM/MM-MFEP) method. ${ }^{49}$ In this method, the thermodynamics of the entire system is simplified by using the PMF surface of the QM portion of the system, which enables the simultaneous optimization of a reaction path on the QM PMF surface and the accurate calculation of the free energies of the reaction process. Accurate $a b$ initio QM optimizations can therefore be performed in the conformational ensemble of the MM subsystem.

The QM/MM-MFEP method is well-suited for the study of reaction processes in complicated systems because many MM conformations contribute to the determination of the QM PMF. QM/MM-MFEP is efficient because it uses a minimal number of QM energy and force evaluations to obtain a reaction path. Even so, in order to obtain the QM PMF and associated forces that are accurate enough for reaction path optimizations, the previous implementation requires converged statistical sampling of the MM subsystem associated with each energy and force evaluation of the QM PMF. While the number of QM calculations is minimal in principle, much like a geometry optimization of a pure QM system, converged sampling of the fluctuating MM environment for each QM conformation can be extremely demanding in terms of computational resources. In addition, because the QM optimization aims to move on the converged PMF surface, the statistical uncertainty in the free energy and its gradients can significantly slow down the QM optimization algorithm. This is precisely the issue we seek to address and overcome in the present work.

Herein, we report two major improvements in the efficiency and accuracy of the QM/MM-MFEP method. First, sequential sampling and optimization of the QM PMF is introduced to significantly reduce the amount of sampling of the MM configuration space required for the optimization on the QM PMF surface. Second, a polarizable QM charge model is also implemented to provide improved accuracy for the QM PMF and gradient calculations. The sequential sampling and optimization strategy developed here for the QM/ MM-MFEP method is similar in nature to the iterative optimization of the QM/MM total energy developed in the QM/ 
MM-FE approach. ${ }^{21}$ We have applied the iterative QM/MMMFEP method to two reaction processes: the $\mathrm{S}_{\mathrm{N}} 2$ reaction of $\mathrm{Cl}^{-}+\mathrm{CH}_{3} \mathrm{Cl}$ in aqueous solution and the second proton transfer step of the tautomerization reaction catalyzed by the enzyme 4-oxalocrotonate tautomerase (4OT). For both processes, we determined the reaction path on the PMF surface of the QM subsystem, including the structures of the reactant state, transition state, and product state of each reaction. We also calculated the corresponding activation free energies. For comparison, the activation free energy of the $\mathrm{S}_{\mathrm{N}} 2$ reaction of $\mathrm{Cl}^{-}+\mathrm{CH}_{3} \mathrm{Cl}$ was also computed using the umbrella sampling technique with direct $\mathrm{QM} / \mathrm{MM}$ dynamic sampling. The free energies from umbrella sampling and QM/MMMFEP are in good agreement with each other, indicating that the QM/MM-MFEP method is accurate. The results for the 4OT system are also in good agreement with previous results from QM/MM-FE simulations. The results of both reactions demonstrate the potential of this method for simulating chemical processes in solution and in enzymes.

\section{THEORY}

\section{A. Background on the potential of mean force and QM/MM-MFEP}

The progress of a chemical reaction process is often characterized by the structural change of a selected group of atoms. Within the QM/MM context, it is natural to describe the progress of the reaction system, e.g., reactant, product, and transition state, by the conformations of the QM subsystem. Therefore, following the previously developed QM/ MM-MFEP method, we simplify the thermodynamics of the entire system by defining the PMF of a QM/MM system in terms of the QM conformation as

$$
A\left(\boldsymbol{r}_{\mathrm{QM}}\right)=-\frac{1}{\beta} \ln \left[\int \exp \left(-\beta E\left(\boldsymbol{r}_{\mathrm{QM}}, \boldsymbol{r}_{\mathrm{MM}}\right)\right) d \boldsymbol{r}_{\mathrm{MM}}\right],
$$

where $E\left(\boldsymbol{r}_{\mathrm{QM}}, \boldsymbol{r}_{\mathrm{MM}}\right)$ is the total energy of the entire system expressed as a function of the coordinates of the QM and MM subsystems, $\boldsymbol{r}_{\mathrm{QM}}$ and $\boldsymbol{r}_{\mathrm{MM}}$, respectively. The gradient of the PMF, i.e., the free-energy gradient, is then

$$
\frac{\partial A\left(\boldsymbol{r}_{\mathrm{QM}}\right)}{\partial \boldsymbol{r}_{\mathrm{QM}}}=\left\langle\frac{\partial E\left(\boldsymbol{r}_{\mathrm{QM}}, \boldsymbol{r}_{\mathrm{MM}}\right)}{\partial \boldsymbol{r}_{\mathrm{QM}}}\right\rangle_{E, \boldsymbol{r}_{\mathrm{MM}}},
$$

which appears conveniently as the ensemble average of the gradient of the QM atoms, a term that can be obtained from MD simulations of the MM atoms. The notation $\langle X\rangle_{E, r_{M M}}$ indicates an average over the MM degrees of freedom in the ensemble generated from the energy function $E\left(\boldsymbol{r}_{\mathrm{QM}}, \boldsymbol{r}_{\mathrm{MM}}\right)$,

$$
\langle X\rangle_{E, \boldsymbol{r}_{\mathrm{MM}}}=\frac{\int X\left(\boldsymbol{r}_{\mathrm{MM}}\right) \exp \left(-\beta E\left(\boldsymbol{r}_{\mathrm{QM}}, \boldsymbol{r}_{\mathrm{MM}}\right)\right) d \boldsymbol{r}_{\mathrm{MM}}}{\int \exp \left(-\beta E\left(\boldsymbol{r}_{\mathrm{QM}}, \boldsymbol{r}_{\mathrm{MM}}\right)\right) d \boldsymbol{r}_{\mathrm{MM}}} .
$$

Note that Eq. (2) is exact and is independent of the form of the energy function of the target $\mathrm{QM} / \mathrm{MM}$ system. It is also possible to extend this equation to systems beyond a QM/MM description.

Equations (1) and (2) form the foundation for all methods that optimize the molecular conformation on a PMF surface, ${ }^{50-58}$ including the QM/MM-MFEP method. In our method, the optimization of the reaction path is carried out efficiently in a discretized representation. ${ }^{49}$ To reduce the statistical variance and hence achieve better convergence associated with the ensemble average in the calculation of the PMF using Eq. (1), we always use the free-energy perturbation formula for the chain of conformations, ${ }^{21,49}$

$$
\begin{aligned}
A\left(\boldsymbol{r}_{\mathrm{QM}}\right)= & A_{\mathrm{ref}}-\frac{1}{\beta} \ln \left\langle\operatorname { e x p } \left\{-\beta\left[E\left(\boldsymbol{r}_{\mathrm{QM}}, \boldsymbol{r}_{\mathrm{MM}}\right)\right.\right.\right. \\
& \left.\left.\left.-E_{\mathrm{ref}}\left(\boldsymbol{r}_{\mathrm{MM}}\right)\right]\right\}\right\rangle_{E_{\mathrm{ref}} \boldsymbol{r}_{\mathrm{MM}}} .
\end{aligned}
$$

Here, $A_{\text {ref }}$ is the PMF of a reference QM conformation, and $E_{\text {ref }}\left(\boldsymbol{r}_{\mathrm{MM}}\right)$ is the energy of the reference QM conformation as a function of the MM conformation. $E_{\text {ref }}\left(\boldsymbol{r}_{\mathrm{MM}}\right)$ is normally chosen to be $E\left(r_{\mathrm{QM}}^{\mathrm{ref}}, \boldsymbol{r}_{\mathrm{MM}}\right)$, the QM/MM total energy of the system at a reference QM geometry $\boldsymbol{r}_{\mathrm{QM}}^{\mathrm{ref}}$,

$$
E_{\mathrm{ref}}\left(\boldsymbol{r}_{\mathrm{MM}}\right)=E\left(\boldsymbol{r}_{\mathrm{QM}}^{\mathrm{ref}}, \boldsymbol{r}_{\mathrm{MM}}\right) \text {. }
$$

The PMF gradient associated with Eq. (4) is then

$$
\frac{\partial A\left(\boldsymbol{r}_{\mathrm{QM}}\right)}{\partial \boldsymbol{r}_{\mathrm{QM}}}=\frac{\left\langle\frac{\partial E\left(\boldsymbol{r}_{\mathrm{QM}}, \boldsymbol{r}_{\mathrm{MM}}\right)}{\partial \boldsymbol{r}_{\mathrm{QM}}} \exp \left[-\beta\left(E\left(\boldsymbol{r}_{\mathrm{QM}}, \boldsymbol{r}_{\mathrm{MM}}\right)-E_{\mathrm{ref}}\left(\boldsymbol{r}_{\mathrm{MM}}\right)\right)\right]\right\rangle_{E_{\mathrm{re}}, \boldsymbol{r}_{\mathrm{MM}}}}{\left\langle\exp \left[-\beta\left(E\left(\boldsymbol{r}_{\mathrm{QM}}, \boldsymbol{r}_{\mathrm{MM}}\right)-E_{\mathrm{ref}}\left(\boldsymbol{r}_{M M}\right)\right)\right]\right\rangle_{E_{\mathrm{ref}}, \boldsymbol{r}_{\mathrm{MM}}}}
$$

Using the PMF and its gradient given by Eqs. (4) and (6), we can carry out the optimization of a QM conformation along the reaction path according to

$\min _{r_{\mathrm{QM}}} A\left(\boldsymbol{r}_{\mathrm{QM}}\right)=\min _{r_{\mathrm{QM}}}\left\{A_{\mathrm{ref}}-\frac{1}{\beta} \ln \left\langle\exp \left[-\beta\left(E-E_{\mathrm{ref}}\right)\right]\right\rangle_{E_{\mathrm{re}}, \boldsymbol{r}_{\mathrm{MM}}}\right\}$.

Because the QM degrees of freedom are coupled with the
MM degrees of freedom, a straightforward minimization algorithm requires each step in the optimization of the QM conformations to be associated with the converged sampling of the MM ensemble.

In this optimization scheme, every QM optimization step to a new conformation on the PMF surface is followed by a course of MD sampling of the MM conformations, usually with a simulation time of $100-1000$ ps. Such extensive MM sampling is required so that the QM PMF and gradient 
obtained from Eqs. (4) and (6) are sufficiently accurate for successful structure and reaction path optimization of the QM subsystem. ${ }^{49,56,58}$

While the QM/MM-MFEP method has been successfully applied to the proton transfer reaction in triosephosphate isomerase (TIM), in practical simulations this method is still limited by two factors. First, 100-1000 ps of MD simulations on a system of $\sim 10000$ atoms is expensive. Even worse is that such MD simulations must be repeated for each step in the optimization process of the QM subsystem. Additionally, the intrinsic fluctuations and limited simulation times for the MD sampling lead to slow convergence in the path optimization if a new MD simulation is always begun immediately after every QM geometry optimization step. Therefore, the direct optimization approach implemented in previous work becomes inefficient when the QM/MM-MFEP method is applied to larger molecular systems.

If our goal were to accurately evaluate the PMF and its gradient at all the conformations involved in the path optimizations, then there might be no way around the converged sampling of the MM subsystem carried out each time a new point on the PMF is visited. However, our goal is to find the relevant stationary points on the PMF that comprise the MFEP of the reaction process. Exploring this approach opens up new possibilities and leads to greatly enhanced efficiency. Our development of the reaction path optimization on the $\mathrm{PMF}$, presented here in the QM/MM context, is also generally applicable to the optimization of PMF degrees of freedom other than the QM conformations.

\section{B. QM/MM-FE: Sequential QM and MM optimization on the $Q M / M M$ potential energy surface}

To set the stage for the presentation of our new method, let us first review the development of our previous QM/ MM-FE approach whose framework shares great similarity with the current work. ${ }^{21,24,40}$ In this approach, one aims to find the minimum energy reaction path by performing the following minimization:

$$
\min _{\boldsymbol{r}_{\mathrm{QM}}, \boldsymbol{r}_{\mathrm{MM}}} E\left(\boldsymbol{r}_{\mathrm{QM}}, \boldsymbol{r}_{\mathrm{MM}}\right) .
$$

A straightforward realization of this goal would be the concurrent optimization of both $\boldsymbol{r}_{\mathrm{QM}}$ and $\boldsymbol{r}_{\mathrm{MM}}$. For this purpose, one must perform a QM calculation at each step in the optimization process to compute the exact energy and gradient, which is expensive for ab initio QM methods.

In general optimization problems, ${ }^{59}$ it is known that in certain cases it may be more desirable to break up a process into an iterative sequence of two (or more) steps as follows: (i) optimize a subset of the system, $\boldsymbol{A}$, with the rest of the system, $\boldsymbol{B}$, held constant; (ii) optimize $\boldsymbol{B}$ with $\boldsymbol{A}$ fixed according to the results obtained in step (i). Thus, in the QM/ MM-FE method, instead of a concurrent optimization of the QM and MM degrees of freedom, Zhang, Liu, and Yang ${ }^{21}$ developed an iterative sequential optimization protocol that has proven to be quite effective in reducing the number of QM energy and gradient evaluations. The main idea is this: Starting from a given structure of the QM/MM molecular system, the optimization is separated into two processes. One first optimizes $\boldsymbol{r}_{\mathrm{QM}}$ with fixed $\boldsymbol{r}_{\mathrm{MM}}$, which is at an approximate minimum in the MM degrees of freedom. Afterwards, the conformation of the MM subsystem, $\boldsymbol{r}_{\mathrm{MM}}$, is optimized with $\boldsymbol{r}_{\mathrm{QM}}$ fixed. To reduce the number of QM evaluations, an approximate QM/MM total energy, $\widetilde{E}\left(\boldsymbol{r}_{\mathrm{QM}}, \boldsymbol{r}_{\mathrm{MM}}\right)$, is used for the MM optimization. The electrostatic interactions between the QM and MM atoms are approximated by the Coulombic interactions between the point charges of the MM atoms and the electrostatic potential (ESP) fitted charges of the QM atoms. This process is then iterated until convergence, which is normally achieved within a few iterations (often less than ten). Expressed as an algorithm, the Zhang-Liu-Yang $\mathrm{QM} / \mathrm{MM}$ optimization procedure is as follows:

Algorithm 1: Sequential QM and MM optimization on the $Q M / M M$ potential energy surface ${ }^{21}$

(1) Initiate a structure of the QM subsystem, $\boldsymbol{r}_{\mathrm{QM}}^{(0)}$ and set $n=0$;

(2) Set $n=n+1$;

(a) Carry out an MM minimization with QM atoms fixed at $\boldsymbol{r}_{\mathrm{QM}}^{(n-1)}$,

$\boldsymbol{r}_{\mathrm{MM}}^{(n)}=\underset{r_{\mathrm{MM}}}{\arg \min } \widetilde{E}\left(\boldsymbol{r}_{\mathrm{QM}}^{(n-1)}, \boldsymbol{r}_{\mathrm{MM}}\right)$.

(b) Carry out a QM optimization with MM atoms fixed at $\boldsymbol{r}_{\mathrm{MM}}^{(n)}$,

$\boldsymbol{r}_{\mathrm{QM}}^{(n)}=\arg \min _{r_{\mathrm{QM}}} E\left(\boldsymbol{r}_{\mathrm{QM}}, \boldsymbol{r}_{\mathrm{MM}}^{(n)}\right)$.

(3) Go to step (2) until converged.

\section{QM/MM-MFEP: Sequential sampling and optimization on the QM PMF surface}

We can also cast the problem of optimization on the QM $\mathrm{PMF}$ as a simultaneous optimization in $\boldsymbol{r}_{\mathrm{QM}}$, the QM degrees of freedom, and $\rho\left(\boldsymbol{r}_{\mathrm{MM}}\right)$, the configuration distribution of the MM degrees of freedom. ${ }^{49}$ Specifically, the MD sampling of the MM degrees of freedom can be viewed as an optimization of $\rho\left(r_{\mathrm{MM}}\right), 4$

$$
\begin{aligned}
A\left(\boldsymbol{r}_{\mathrm{QM}}\right)= & -\frac{1}{\beta} \ln \left[\int \exp \left(-\beta E\left(\boldsymbol{r}_{\mathrm{QM}}, \boldsymbol{r}_{\mathrm{MM}}\right)\right) d \boldsymbol{r}_{\mathrm{MM}}\right] \\
= & \min _{\rho\left(\boldsymbol{r}_{\mathrm{MM}}\right)} \int \rho\left(\boldsymbol{r}_{\mathrm{MM}}\right)\left[E\left(\boldsymbol{r}_{\mathrm{QM}}, \boldsymbol{r}_{\mathrm{MM}}\right)\right. \\
& \left.+\frac{1}{\beta} \ln \rho\left(\boldsymbol{r}_{\mathrm{MM}}\right)\right] d \boldsymbol{r}_{\mathrm{MM}},
\end{aligned}
$$

and

$$
\begin{aligned}
\min _{\boldsymbol{r}_{\mathrm{QM}}} A\left(\boldsymbol{r}_{\mathrm{QM}}\right)= & \min _{\boldsymbol{r}_{\mathrm{QM}}} \min _{\rho\left(\boldsymbol{r}_{\mathrm{MM}}\right)} \int \rho\left(\boldsymbol{r}_{\mathrm{MM}}\right)\left[E\left(\boldsymbol{r}_{\mathrm{QM}}, \boldsymbol{r}_{\mathrm{MM}}\right)\right. \\
& \left.+\frac{1}{\beta} \ln \rho\left(\boldsymbol{r}_{\mathrm{MM}}\right)\right] d \boldsymbol{r}_{\mathrm{MM}} .
\end{aligned}
$$

Like the QM/MM-FE method, we can improve the optimization efficiency by reformulating the concurrent optimization of the QM subsystem and the statistical sampling of the MM subsystem into an iterative step of sequential MD sam- 
pling of the MM system at a fixed QM structure and subsequent optimization of the QM subsystem within the fixed MM conformational ensemble. The approximate energy function model, $\widetilde{E}\left(\boldsymbol{r}_{\mathrm{QM}}, \boldsymbol{r}_{\mathrm{MM}}\right)$, plays a crucial role in reducing the number of QM energy and gradient evaluations required. In the MD sampling of the MM conformations, $\widetilde{E}\left(\boldsymbol{r}_{\mathrm{QM}}, \boldsymbol{r}_{\mathrm{MM}}\right)$ acts as a reference sampling energy function, $E_{\mathrm{ref}}\left(\boldsymbol{r}_{\mathrm{MM}}\right)$, to drive the motion of the MM atoms without performing a QM calculation at every MD step. That is, $E_{\mathrm{ref}}\left(\boldsymbol{r}_{\mathrm{MM}}\right)$ $=\widetilde{E}\left(r_{\mathrm{QM}}^{\mathrm{ref}}, \boldsymbol{r}_{\mathrm{MM}}\right)$, the QM/MM total energy of the system derived from a reference QM geometry $\boldsymbol{r}_{\mathrm{QM}}^{\mathrm{ref}}$, similar to that in Eq. (5). In the optimization of the QM subsystem within the fixed-size MM conformational ensemble, $\widetilde{E}\left(\boldsymbol{r}_{\mathrm{QM}}, \boldsymbol{r}_{\mathrm{MM}}\right)$ is again used to avoid QM calculations for each new conformation in the MM ensemble. Further discussion of $\widetilde{E}\left(\boldsymbol{r}_{\mathrm{QM}}, \boldsymbol{r}_{\mathrm{MM}}\right)$ will be given in Sec. II D.

Expressed as an algorithm, the sequential sampling and optimization method is as follows:

Algorithm 2: Sequential sampling and optimization on the $Q M / M M P M F$ surface

(1) Initiate a structure of the QM subsystem, $\boldsymbol{r}_{\mathrm{QM}}^{(0)}$, and set $n=0$.

(2) Set $n=n+1$;

(a) Carry out MD sampling of the MM ensemble with QM atoms fixed at $\boldsymbol{r}_{\mathrm{QM}}^{(n-1)}$,

$\left\{\boldsymbol{r}_{\mathrm{MM}}^{(n)}(\tau), \quad \tau=1, \ldots, N\right\}$

$\Leftarrow \mathrm{MD}$ sampling based on $E_{\mathrm{ref}}\left(\boldsymbol{r}_{\mathrm{MM}}\right)$,

where $\tau$ is the step of the MD simulation, $N$ is the number of MD steps, the reference energy $E_{\text {ref }}\left(\boldsymbol{r}_{\mathrm{MM}}\right)$ is

$E_{\mathrm{ref}}\left(\boldsymbol{r}_{\mathrm{MM}}\right)=\tilde{E}\left(\boldsymbol{r}_{\mathrm{QM}}^{\mathrm{ref}(n-1)}, \boldsymbol{r}_{\mathrm{MM}}\right)$

and the reference QM structure $\boldsymbol{r}_{\mathrm{QM}}^{\mathrm{ref}(n-1)}$ is derived from $\boldsymbol{r}_{\mathrm{QM}}^{(n-1)}$, the QM geometry from the previous iteration.

(b) Carry out a QM optimization with the MM ensemble fixed at $\left\{\boldsymbol{r}_{\mathrm{MM}}^{(n)}(\tau)\right\}$,

$\boldsymbol{r}_{\mathrm{QM}}^{(n)}=\underset{r_{\mathrm{QM}}}{\arg \min } A^{(n)}\left(\boldsymbol{r}_{\mathrm{QM}}\right)$,

where the object of minimization is the QM PMF (or QM free energy) in the $n$th iteration given by a finite sum representation of Eq. (4),

$$
\begin{aligned}
A^{(n)}\left(\boldsymbol{r}_{\mathrm{QM}}\right)= & A_{\mathrm{ref}} \\
& -\frac{1}{\beta} \ln \left\{\frac { 1 } { N } \sum _ { \tau = 1 } ^ { N } \operatorname { e x p } \left\{-\beta\left[\widetilde{E}\left(\boldsymbol{r}_{\mathrm{QM}}, \boldsymbol{r}_{\mathrm{MM}}^{(n)}(\tau)\right)\right.\right.\right. \\
& \left.\left.\left.-E_{\mathrm{ref}}\left(\boldsymbol{r}_{\mathrm{MM}}^{(n)}(\tau)\right)\right]\right\}\right\} .
\end{aligned}
$$

The corresponding gradient with respect to the $i$ th QM coordinate is given by the finite sum

$\frac{\partial A^{(n)}\left(\boldsymbol{r}_{\mathrm{QM}}\right)}{\partial \boldsymbol{r}_{\mathrm{QM}, i}}=\frac{\sum_{\tau=1}^{N} \partial \widetilde{E}\left(\boldsymbol{r}_{\mathrm{QM}}, \boldsymbol{r}_{\mathrm{MM}}^{(n)}(\tau)\right) / \partial \boldsymbol{r}_{\mathrm{QM}, i} \exp \left\{-\beta\left[\widetilde{E}\left(\boldsymbol{r}_{\mathrm{QM}}, \boldsymbol{r}_{\mathrm{MM}}^{(n)}(\tau)\right)-E_{\mathrm{ref}}\left(\boldsymbol{r}_{\mathrm{MM}}^{(n)}(\tau)\right)\right]\right\}}{\sum_{\tau=1}^{N} \exp \left\{-\beta\left[\tilde{E}\left(\boldsymbol{r}_{\mathrm{QM}}, \boldsymbol{r}_{\mathrm{MM}}^{(n)}(\tau)\right)-E_{\mathrm{ref}}\left(\boldsymbol{r}_{\mathrm{MM}}^{(n)}(\tau)\right)\right]\right\}}$.

(c) Update the reference structure $\boldsymbol{r}_{\mathrm{QM}}^{\mathrm{ref}(n)}$ based on the minimized $\boldsymbol{r}_{\mathrm{QM}}^{(n)}$.

(3) Go to step (2) until converged.

Note here that step (2) is also termed an optimization "cycle" in later discussions.

In this algorithm, the relative PMF of a given QM conformation is computed by free-energy perturbation with respect to a reference QM conformation. The choice of the reference QM conformation will of course directly affect the accuracy and convergence of the FEP calculations. In our experience, for the optimization of the reactant and product structures, we set the QM structure optimized in the previous step as the reference QM structure; that is, $\boldsymbol{r}_{\mathrm{QM}}^{\mathrm{ref}(n-1)}=\boldsymbol{r}_{\mathrm{QM}}^{(n-1)}$ in Eq. (14). For the optimization of other QM structures along the reaction path, in accordance with the FEP calculations of relative free energies between two adjacent conformations, we use the QM structure from the neighbors $\boldsymbol{r}_{\mathrm{QM}}^{\mathrm{ref}(n-1)}=\boldsymbol{r}_{\mathrm{QM}}^{(n-1)}$ (neighboring state). Further details will be provided in Sec. II E.

The key feature of our new QM/MM-MFEP algorithm is the iterative QM optimization in a fixed MM ensemble, Eq. (15) in Algorithm 2. Because the MM ensemble, $\left\{\boldsymbol{r}_{\mathrm{MM}}^{(n)}(\tau)\right\}, \tau=1, \ldots, N$, is finite and remains fixed throughout the course of the QM optimization for $\boldsymbol{r}_{\mathrm{OM}}^{(n)}$, one can obtain the precise PMF, Eq. (16), and its gradient, Eq. (17), defined within this ensemble. This circumvents the difficult and costly convergence problems associated with MM sampling. The optimization of the PMF can be carried out efficiently using classical numerical optimization tools. Each optimized $\mathrm{QM}$ structure $\boldsymbol{r}_{\mathrm{QM}}^{(n)}$ in turn provides the next reference $\mathrm{QM}$ structure $\boldsymbol{r}_{\mathrm{QM}}^{\mathrm{ref}(n)}$ and its energy function, $E_{\mathrm{ref}}\left(\boldsymbol{r}_{\mathrm{MM}}\right)$ $=\widetilde{E}\left(\boldsymbol{r}_{\mathrm{QM}}^{\mathrm{ref}(n)}, \boldsymbol{r}_{\mathrm{MM}}\right)$, for the next round of MD sampling of the MM conformations. Each optimized QM structure should 
improve on the previous one by providing a better QM geometry and corresponding ESP charges for the MM simulation in the next cycle. As we will show in Sec. IV, Results, our QM/MM-MFEP method converges as efficiently as the QM/MM-FE method.

From the consideration of computational costs, the use of a finite, fixed-size ensemble of MM conformations improves the utilization of the MM conformations and avoids repetitive MD sampling at each step of the QM structure optimization. Thus, instead of performing excessive steps of MD simulation that likely have significant overlap with each other, a few cycles of MD simulation are able to yield converged results in the current method.

To make a clear comparison, we describe here our previous implementation of QM/MM-MFEP (Ref. 49) and other works involving optimization of the free energy ${ }^{56,58}$ as a concurrent MM sampling and QM optimization. Such an approach is shown in the following algorithm:

Algorithm 3: Concurrent sampling and optimization on the QM PMF surface

(1) Initiate a structure of the QM subsystem, $\boldsymbol{r}_{\mathrm{QM}}^{(0)}$ and set $n=0$

(2) Set $n=n+1$;

(a) Carry out MD sampling of the MM ensemble with $\mathrm{QM}$ atoms fixed at $\boldsymbol{r}_{\mathrm{QM}}^{(n-1)}$,

$$
\begin{aligned}
& \left\{\boldsymbol{r}_{\mathrm{MM}}^{(n)}(\tau), \tau=1, \ldots, N\right\} \\
& \quad \Leftarrow \mathrm{MD} \text { sampling based on } E_{\mathrm{ref}}\left(\boldsymbol{r}_{\mathrm{MM}}\right) .
\end{aligned}
$$

As in Algorithm 2, an approximate energy function $\widetilde{E}\left(\boldsymbol{r}_{\mathrm{QM}}, \boldsymbol{r}_{\mathrm{MM}}\right)$ is used to compute the reference energy $E_{\text {ref }}\left(\boldsymbol{r}_{\mathrm{MM}}\right)$. During the MD simulation, the QM PMF and PMF gradient are determined by Eqs. (4) and (6).

(b) Complete one step of movement for the QM optimization using the QM PMF and its gradient computed in (a),

$$
\begin{gathered}
\boldsymbol{r}_{\mathrm{QM}}^{(n)} \Leftarrow \text { one step in the } \mathrm{QM} \text { optimization } \\
\text { based on } A\left(\boldsymbol{r}_{\mathrm{QM}}^{(n-1)}\right) \text { and } \frac{\partial A\left(\boldsymbol{r}_{\mathrm{QM}}^{(n-1)}\right)}{\partial \boldsymbol{r}_{\mathrm{QM}}}
\end{gathered}
$$

(c) Update the reference structure $\boldsymbol{r}_{\mathrm{QM}}^{\mathrm{ref}(n)}$ based on the new $\mathrm{QM}$ structure $\boldsymbol{r}_{\mathrm{QM}}^{(n)}$.

(3) Go to step (2) until converged.

As discussed in Sec. II A, this concurrent sampling and optimization of Eq. (7) requires the accurate and converged value of the PMF and its gradient used in step (2b) shown above. Compared with Algorithm 2, the MM sampling in Eq. (18) is much more frequent, occurring at each new QM structure during the course of the QM optimization.

\section{Approximate total QM/MM energy $\tilde{E}\left(r_{\mathrm{QM}}, r_{\mathrm{MM}}\right)$}

For a system investigated using the combined QM/MM method, the total potential energy of the system can be expressed schematically as

$$
\begin{aligned}
E\left(\boldsymbol{r}_{\mathrm{QM}}, \boldsymbol{r}_{\mathrm{MM}}\right)= & E_{\mathrm{QM}}\left(\boldsymbol{r}_{\mathrm{QM}}\right)+E_{\mathrm{QM} / \mathrm{MM}, \mathrm{ele}}\left(\boldsymbol{r}_{\mathrm{QM}}, \boldsymbol{r}_{\mathrm{MM}}\right) \\
& +E_{\mathrm{QM} / \mathrm{MM}, \mathrm{vdw}}\left(\boldsymbol{r}_{\mathrm{QM}}, \boldsymbol{r}_{\mathrm{MM}}\right) \\
& +E_{\mathrm{QM} / \mathrm{MM}, \mathrm{cov}}\left(\boldsymbol{r}_{\mathrm{QM}}, \boldsymbol{r}_{\mathrm{MM}}\right)+E_{\mathrm{MM}}\left(r_{\mathrm{MM}}\right) .
\end{aligned}
$$

The terms on the right-hand side are the QM internal energy, the electrostatic interactions between the QM and MM subsystems, the van der Waals interactions between the QM and MM subsystems, covalent interactions between the QM and MM subsystems, and the interactions of the MM subsystem, respectively. The first two terms on the right-hand side must be solved together in a self-consistent manner to capture the correct polarization interactions of the QM atoms. That is,

$$
E_{\mathrm{QM}}\left(\boldsymbol{r}_{\mathrm{QM}}\right)+E_{\mathrm{QM} / \mathrm{MM}, \mathrm{ele}}\left(\boldsymbol{r}_{\mathrm{QM}}, \boldsymbol{r}_{\mathrm{MM}}\right)=\left\langle\Psi\left|H_{\mathrm{eff}}\right| \Psi\right\rangle,
$$

in which $H_{\text {eff }}$ is the effective QM Hamiltonian including the Coulombic potential term from the MM atoms.

The derivative of Eq. (20), required for the PMF gradient calculation in Eqs. (2), (6), and (17), is

$$
\frac{\partial E\left(\boldsymbol{r}_{\mathrm{QM}}, \boldsymbol{r}_{\mathrm{MM}}\right)}{\partial \boldsymbol{r}_{\mathrm{QM}, i}}=\frac{\partial\left(E_{\mathrm{QM}}\left(\boldsymbol{r}_{\mathrm{QM}}\right)+E_{\mathrm{QM} / \mathrm{MM}, \mathrm{ele}}\left(\boldsymbol{r}_{\mathrm{QM}}, \boldsymbol{r}_{\mathrm{MM}}\right)\right)}{\partial \boldsymbol{r}_{\mathrm{QM}, i}}+\frac{\partial\left(E_{\mathrm{QM} / \mathrm{MM}, \mathrm{vdw}}\left(\boldsymbol{r}_{\mathrm{QM}}, \boldsymbol{r}_{\mathrm{MM}}\right)+E_{\mathrm{QM} / \mathrm{MM}, \mathrm{cov}}\left(\boldsymbol{r}_{\mathrm{QM}}, \boldsymbol{r}_{\mathrm{MM}}\right)+E_{\mathrm{MM}}\left(\boldsymbol{r}_{\mathrm{MM}}\right)\right)}{\partial \boldsymbol{r}_{\mathrm{QM}, i}} .
$$

The first term on the right, based on the Hellman-Feynman theorem, can be obtained analytically in any standard electronic structure program, while the second term can be easily computed from a classical MM force field. The direct application of Eq. (22) would require a QM calculation at every MD step, which is only possible for relatively small QM subsystems or QM methods at the semiempirical level. ${ }^{50-55}$
To circumvent the high cost of $a b$ initio QM computations that essentially eliminates the possibility of carrying out long timescale simulations, we develop here an approximate energy function model, i.e., $\widetilde{E}\left(\boldsymbol{r}_{\mathrm{QM}}, \boldsymbol{r}_{\mathrm{MM}}\right)$. As shown in previous algorithms, introducing this term can dramatically reduce the number of QM calculations in the optimization or the MD sampling of the MM subsystem. 
Clearly, this energy function must be constructed on the basis of ab initio QM without a significant loss of accuracy, and it must also be computationally affordable. We summarize here possible forms for the term $\widetilde{E}\left(\boldsymbol{r}_{\mathrm{QM}}, \boldsymbol{r}_{\mathrm{MM}}\right)$ used in the three algorithms, with gradually improved accuracy through successive inclusion of higher order interaction terms. In practical applications, the choice of a specific model can be flexibly adjusted depending on the desired level of accuracy.

\section{Simple $Q M$ point charges}

We would like to employ an approximate expression to simplify the calculation of the electrostatic interactions between the QM and MM subsystems, which otherwise must be evaluated using the QM SCF calculations described in Eq. (21). Such an expression must also be readily differentiable to allow the calculation of the gradient of the MM atoms. One approach is to separate the interactions into two components: A QM internal energy and an electrostatic interaction energy between the QM and MM subsystems. We approximate the electrostatic term as the Coulombic interactions between the QM ESP charges and the MM atomic charges, i.e.,

$$
E_{\mathrm{QM} / \mathrm{MM}}^{\mathrm{ESP}}\left(\boldsymbol{r}_{\mathrm{QM}}, \boldsymbol{r}_{\mathrm{MM}}\right)=\sum_{j \in \mathrm{MM}} \sum_{i \in \mathrm{QM}} \frac{q_{j} Q_{i}\left(\boldsymbol{r}_{\mathrm{QM}}, \boldsymbol{r}_{\mathrm{MM}}\right)}{\left|\boldsymbol{r}_{\mathrm{QM}, i}-\boldsymbol{r}_{\mathrm{MM}, j}\right|} .
$$

Here, $Q_{i}\left(\boldsymbol{r}_{\mathrm{QM}}, \boldsymbol{r}_{\mathrm{MM}}\right)$ is the ESP fitted charge of QM atom $i$, and $q_{j}$ is the point charge of MM atom $j$ from the MM force field. While the MM atomic charges are constant in common force fields, the $\mathrm{QM}$ electrostatic charges $\left\{Q_{i}\right\}$ are clearly dependent on both $\boldsymbol{r}_{\mathrm{QM}}$ and $\boldsymbol{r}_{\mathrm{MM}}$. The QM internal energy, $E_{1}\left(\boldsymbol{r}_{\mathrm{QM}}, \boldsymbol{r}_{\mathrm{MM}}\right)$, is defined as ${ }^{21,25}$

$$
E_{1}\left(\boldsymbol{r}_{\mathrm{QM}}, \boldsymbol{r}_{\mathrm{MM}}\right)=\left\langle\Psi\left|H_{\mathrm{eff}}\right| \Psi\right\rangle-E_{\mathrm{QM} / \mathrm{MM}}^{\mathrm{ESP}}\left(\boldsymbol{r}_{\mathrm{QM}}, \boldsymbol{r}_{\mathrm{MM}}\right) .
$$

This QM internal energy is the energy of the QM system in the electrostatic potential of the MM atoms, minus the Coulombic interactions between the QM ESP charges and MM atomic charges. The QM energy $\left\langle\Psi\left|H_{\text {eff }}\right| \Psi\right\rangle$ also depends on both $\boldsymbol{r}_{\mathrm{QM}}$ and $\boldsymbol{r}_{\mathrm{MM}}$. The QM/MM total energy of Eq. (20) can then be expressed as

$$
\begin{aligned}
E\left(\boldsymbol{r}_{\mathrm{QM}}, \boldsymbol{r}_{\mathrm{MM}}\right)= & E_{1}\left(\boldsymbol{r}_{\mathrm{QM}}, \boldsymbol{r}_{\mathrm{MM}}\right)+\sum_{j \in \mathrm{MM}} \sum_{i \in \mathrm{QM}} \frac{q_{j} Q_{i}\left(\boldsymbol{r}_{\mathrm{QM}}, \boldsymbol{r}_{\mathrm{MM}}\right)}{\left|\boldsymbol{r}_{\mathrm{QM}, i}-\boldsymbol{r}_{\mathrm{MM}, j}\right|} \\
& +E_{\mathrm{QM} / \mathrm{MM}, \mathrm{vdw}}\left(\boldsymbol{r}_{\mathrm{QM}}, \boldsymbol{r}_{\mathrm{MM}}\right) \\
& +E_{\mathrm{QM} / \mathrm{MM}, \mathrm{cov}}\left(\boldsymbol{r}_{\mathrm{QM}}, \boldsymbol{r}_{\mathrm{MM}}\right)+E_{\mathrm{MM}}\left(\boldsymbol{r}_{\mathrm{MM}}\right) .
\end{aligned}
$$

Obviously, the term $E_{\mathrm{QM} / \mathrm{MM}}^{\mathrm{ESP}}\left(\boldsymbol{r}_{\mathrm{QM}}, \boldsymbol{r}_{\mathrm{MM}}\right)$ captures the essence of the electrostatic interactions between the QM and $\mathrm{MM}$ subsystems. The significance of this QM ESP charge expression is that it provides us great flexibility as the interactions are now expressed in a classical pairwise MM form. To improve the accuracy of the $\mathrm{QM} / \mathrm{MM}$ electrostatic interactions, we can approximate the polarization in $Q_{i}\left(\boldsymbol{r}_{\mathrm{QM}}, \boldsymbol{r}_{\mathrm{MM}}\right)$ with an explicit analytic dependence on $\boldsymbol{r}_{\mathrm{QM}}$ and $\boldsymbol{r}_{\mathrm{MM}}$, and/or add electrostatic multipoles to each atom or bond.

To develop various approximations to the total energy, we use the classical perturbation-response model commonly employed in quantum mechanics. For this purpose, we need a reference state for both the QM and MM subsystems upon which we can make expansions of the QM/MM total energy. The optimal choice of the reference MM system has been discussed in our previous work. ${ }^{49}$ Here we recapitulate the method. The MM electrostatic potential at position $r$ and at any time $\tau$ in the MD simulation is given by the expression

$$
v_{\mathrm{MM}}(\boldsymbol{r})=\sum_{j=1}^{M_{n}} \frac{q_{j}(\tau)}{\left|\boldsymbol{r}-\boldsymbol{r}_{\mathrm{MM}, j}(\tau)\right|},
$$

where $M_{n}$ is the number of MM atoms, $\boldsymbol{r}_{\mathrm{MM}, j}(\tau)$ is the position vector of MM atom $j$ at time $\tau$, and $q_{j}(\tau)$ is the atomic charge of MM atom $j$ at the $\pi$ th snapshot. Again, the last term is a constant if a nonpolarizable MM force field is used as in the current work. The electrostatic potential $v_{\mathrm{MM}}(\boldsymbol{r})$ enters into the QM calculations to determine $E_{1}\left(\boldsymbol{r}_{\mathrm{QM}}, \boldsymbol{r}_{\mathrm{MM}}\right)$ and fluctuates in the MD ensemble. To describe this fluctuation, we choose a reference potential as the mean field of the MM environment. ${ }^{49}$ Namely, the averaged electrostatic potential at $\boldsymbol{r}$ is

$$
v_{\mathrm{MM}}^{0}(\boldsymbol{r})=\frac{1}{N} \sum_{\tau=1}^{N} \sum_{j=1}^{M_{n}} \frac{q_{j}(\tau)}{\left|\boldsymbol{r}-\boldsymbol{r}_{\mathrm{MM}, j}(\tau)\right|} .
$$

Here, $N$ is the number of snapshots recorded during an MD simulation. The electrostatic potential $v_{\mathrm{MM}}^{0}(\boldsymbol{r})$ is used in QM calculations to determine $E_{1}\left(r_{\mathrm{QM}}, r_{\mathrm{MM}}^{0}\right)$ in Eq. (28) shown below. In the implementation of Algorithms 2 and 3, the reference mean-field electrostatic potential $v_{\mathrm{MM}}^{0}(\boldsymbol{r})$ is calculated from the previous iteration, as it is required to generate the ensemble for the current iteration.

As a zero-order approximation, we assume that the QM internal energy $E_{1}\left(\boldsymbol{r}_{\mathrm{QM}}, \boldsymbol{r}_{\mathrm{MM}}^{0}\right)$ and $Q_{i}\left(\boldsymbol{r}_{\mathrm{QM}}, \boldsymbol{r}_{\mathrm{MM}}^{0}\right)$, both obtained from a QM calculation with a reference MM conformation $\boldsymbol{r}_{\mathrm{MM}}^{0}$, are constant and generally correct in spite of the fluctuation of the MM environments. That is to say, we approximate the QM internal energy as

$$
E_{1}\left(\boldsymbol{r}_{\mathrm{QM}}, \boldsymbol{r}_{M M}\right) \approx E_{1}\left(\boldsymbol{r}_{\mathrm{QM}}, \boldsymbol{r}_{\mathrm{MM}}^{0}\right),
$$

and the electrostatic interaction as

$$
E_{\mathrm{QM} / \mathrm{MM}}^{\mathrm{ESP}}\left(\boldsymbol{r}_{\mathrm{QM}}, \boldsymbol{r}_{\mathrm{MM}}\right)=\sum_{j \in \mathrm{MM}} \sum_{i \in \mathrm{QM}} \frac{q_{j} Q_{i}\left(\boldsymbol{r}_{\mathrm{QM}}, \boldsymbol{r}_{\mathrm{MM}}^{0}\right)}{\left|\boldsymbol{r}_{\mathrm{QM}, i}-\boldsymbol{r}_{\mathrm{MM}, j}\right|} .
$$

The approximate $\mathrm{QM} / \mathrm{MM}$ total energy is

$$
\begin{aligned}
\widetilde{E}\left(\boldsymbol{r}_{\mathrm{QM}}, \boldsymbol{r}_{\mathrm{MM}}\right)= & E_{1}\left(\boldsymbol{r}_{\mathrm{QM}}, \boldsymbol{r}_{\mathrm{MM}}^{0}\right)+\sum_{j \in \mathrm{MM}} \sum_{i \in \mathrm{QM}} \frac{q_{j} Q_{i}\left(\boldsymbol{r}_{\mathrm{QM}}, \boldsymbol{r}_{\mathrm{MM}}^{0}\right)}{\left|\boldsymbol{r}_{\mathrm{QM}, i}-\boldsymbol{r}_{\mathrm{MM}, j}\right|} \\
& +E_{\mathrm{QM} / \mathrm{MM}, \mathrm{vdw}}\left(\boldsymbol{r}_{\mathrm{QM}}, \boldsymbol{r}_{\mathrm{MM}}\right) \\
& +E_{\mathrm{QM} / \mathrm{MM}, \mathrm{cov}}\left(\boldsymbol{r}_{\mathrm{QM}}, \boldsymbol{r}_{\mathrm{MM}}\right)+E_{\mathrm{MM}}\left(\boldsymbol{r}_{\mathrm{MM}}\right) .
\end{aligned}
$$

Here, we use the electrostatic interaction energy $E_{\mathrm{QM} / \mathrm{MM}}^{\mathrm{ESP}}\left(\boldsymbol{r}_{\mathrm{QM}}, \boldsymbol{r}_{\mathrm{MM}}\right)$ function twice in two different ways. In the expression of the internal energy $E_{1}\left(r_{\mathrm{QM}}, r_{\mathrm{MM}}^{0}\right)$, it enters as a constant, 


$$
E_{\mathrm{QM} / \mathrm{MM}}^{\mathrm{ESP}}\left(\boldsymbol{r}_{\mathrm{QM}}, \boldsymbol{r}_{\mathrm{MM}}^{0}\right)=\sum_{j \in \mathrm{MM}} \sum_{i \in \mathrm{QM}} \frac{q_{j} Q_{i}\left(\boldsymbol{r}_{\mathrm{QM}}, \boldsymbol{r}_{\mathrm{MM}}^{0}\right)}{\left|\boldsymbol{r}_{\mathrm{QM}, i}-\boldsymbol{r}_{\mathrm{MM}, j}^{0}\right|},
$$

independent of $\boldsymbol{r}_{\mathrm{MM}}$. In the approximate total energy of Eq. (30), $E_{\mathrm{QM} / \mathrm{MM}}^{\mathrm{ESP}}\left(\boldsymbol{r}_{\mathrm{QM}}, \boldsymbol{r}_{\mathrm{MM}}\right)$ enters as Eq. (29), the electrostatic interaction energy between the constant QM charges and the MM point charges.

Of course, approximating the complex QM/MM electrostatic interactions in a form that employs simple constant QM ESP charges implies an important assumption, i.e., the variation of the polarization of the QM subsystem due to different MM environments is negligible. Although it may lead to limited precision, the approximate ESP expression performs quite accurately, as shown by work done in our group and by others. ${ }^{20,21,23,24,27,40,42,46,60}$

\section{QM point charges with polarization due to the MM atoms}

The QM internal energy defined in Eq. (24) allows systematic improvements in the accuracy of the approximate QM/MM energy beyond Eq. (30), including polarization effects in the calculation of the terms $E_{1}\left(\boldsymbol{r}_{\mathrm{QM}}, \boldsymbol{r}_{\mathrm{MM}}\right)$ and $E_{\mathrm{QM} / \mathrm{MM}}^{\mathrm{ESP}}\left(\boldsymbol{r}_{\mathrm{QM}}, \boldsymbol{r}_{\mathrm{MM}}\right)$. In our MFEP method, the QM atoms do not move during the MD simulations. Therefore, the interactions responsible for the change in the QM/MM interaction energy and thus necessitating a QM calculation at each MD step, originate from the polarization of the QM atoms in the fluctuating external MM potential of the moving MM atoms. Instead of performing costly, direct QM calculations, we can account for the polarization effects from the fluctuating MM environments with the polarizable QM ESP charge model, ${ }^{25,61}$ which is given by

$$
\begin{aligned}
Q_{i}\left(\boldsymbol{r}_{\mathrm{QM}}, v_{\mathrm{MM}}\right)= & Q_{\mathrm{QM}, i}^{0} \\
& +\sum_{j \in \mathrm{QM}} \chi_{i j}\left[v_{\mathrm{MM}}\left(\boldsymbol{r}_{\mathrm{QM}, j}\right)-v_{\mathrm{MM}}^{0}\left(\boldsymbol{r}_{\mathrm{QM}, j}\right)\right] .
\end{aligned}
$$

Here, $Q_{\mathrm{QM}, i}^{0}=Q_{i}\left(\boldsymbol{r}_{\mathrm{QM}}, v_{\mathrm{MM}}^{0}\right)$ is the reference ESP charge of
QM atom $i$ obtained from a QM calculation with a reference MM electrostatic potential, $v_{\mathrm{MM}}^{0}\left(\boldsymbol{r}_{\mathrm{QM}}\right)$. The term $v_{\mathrm{MM}}\left(\boldsymbol{r}_{\mathrm{QM}, j}\right)$ represents the MM electrostatic potential on QM atom $j$ at position $\boldsymbol{r}_{\mathrm{QM}, j}$, as defined in Eq. (26). Note here that for clarity the MM electrostatic potentials are used as variables instead of the explicit MM coordinates $\boldsymbol{r}_{\mathrm{MM}}$ in $E_{1}\left(\boldsymbol{r}_{\mathrm{QM}}, v_{\mathrm{MM}}\right)$ and $E_{\mathrm{QM} / \mathrm{MM}}^{\mathrm{ESP}}\left(\boldsymbol{r}_{\mathrm{QM}}, v_{\mathrm{MM}}\right)$, which are equivalent to $E_{1}\left(\boldsymbol{r}_{\mathrm{QM}}, \boldsymbol{r}_{\mathrm{MM}}\right)$ and $E_{\mathrm{QM} / \mathrm{MM}}^{\mathrm{ESP}}\left(\boldsymbol{r}_{\mathrm{QM}}, \boldsymbol{r}_{\mathrm{MM}}\right)$, respectively. A response kernel is used to characterize the change of a specific QM ESP charge due to the fluctuation of the external MM electrostatic field. The response kernel is defined as ${ }^{25,61,62}$

$$
\chi_{i j}=\left(\frac{\partial Q_{i}}{\partial V_{\mathrm{MM}}\left(r_{\mathrm{QM}, j}\right)}\right)_{N} .
$$

With the polarization effects included, the QM internal energy becomes ${ }^{25}$

$$
\begin{aligned}
E_{1}\left(\boldsymbol{r}_{\mathrm{QM}}, v_{\mathrm{MM}}\right)= & E_{1}\left(\boldsymbol{r}_{\mathrm{QM}}, v_{\mathrm{MM}}^{0}\right)-\sum_{i \in \mathrm{QM}} \sum_{j \in \mathrm{QM}} \chi_{i j} v_{\mathrm{MM}}^{0}\left(\boldsymbol{r}_{\mathrm{QM}, i}\right) \\
& \times\left[v_{\mathrm{MM}}\left(\boldsymbol{r}_{\mathrm{QM}, j}\right)-v_{\mathrm{MM}}^{0}\left(\boldsymbol{r}_{\mathrm{QM}, j}\right)\right] \\
& -\frac{1}{2} \sum_{i \in \mathrm{QM}} \sum_{j \in \mathrm{QM}}\left[v_{\mathrm{MM}}\left(\boldsymbol{r}_{\mathrm{QM}, i}\right)\right. \\
& \left.-v_{\mathrm{MM}}^{0}\left(\boldsymbol{r}_{\mathrm{QM}, i}\right)\right] \chi_{i j}\left[v_{\mathrm{MM}}\left(\boldsymbol{r}_{\mathrm{QM}, j}\right)\right. \\
& \left.-v_{\mathrm{MM}}^{0}\left(\boldsymbol{r}_{\mathrm{QM}, j}\right)\right] .
\end{aligned}
$$

At the same order of approximation, the electrostatic interaction energy between the QM and MM subsystems is

$$
\begin{aligned}
E_{\mathrm{QM} / \mathrm{MM}}^{\mathrm{ESP}}\left(\boldsymbol{r}_{\mathrm{QM}}, v_{\mathrm{MM}}\right)= & \sum_{i \in \mathrm{QM}} Q_{i}\left(\boldsymbol{r}_{\mathrm{QM}}, v_{\mathrm{MM}}\right) v_{\mathrm{MM}}\left(\boldsymbol{r}_{\mathrm{QM}, i}\right) \\
= & \sum_{i \in \mathrm{QM}}\left[Q_{\mathrm{QM}, i}^{0}+\sum_{j \in \mathrm{QM}} \chi_{i j}\left[v_{\mathrm{MM}}\left(\boldsymbol{r}_{\mathrm{QM}, j}\right)\right.\right. \\
& \left.\left.-v_{\mathrm{MM}}^{0}\left(\boldsymbol{r}_{\mathrm{QM}, j}\right)\right]\right] v_{\mathrm{MM}}\left(\boldsymbol{r}_{\mathrm{QM}, i}\right)
\end{aligned}
$$

The total QM/MM energy is now

$$
\begin{aligned}
\tilde{E}\left(\boldsymbol{r}_{\mathrm{QM}}, \boldsymbol{r}_{\mathrm{MM}}\right)= & E_{1}\left(\boldsymbol{r}_{\mathrm{QM}}, v_{\mathrm{MM}}\right)+E_{\mathrm{QM} / \mathrm{MM}}^{\mathrm{ESP}}\left(\boldsymbol{r}_{\mathrm{QM}}, v_{\mathrm{MM}}\right)+E_{\mathrm{QM} / \mathrm{MM}, \mathrm{vdw}}\left(\boldsymbol{r}_{\mathrm{QM}}, \boldsymbol{r}_{\mathrm{MM}}\right)+E_{\mathrm{QM} / \mathrm{MM}, \mathrm{cov}}\left(\boldsymbol{r}_{\mathrm{QM}}, \boldsymbol{r}_{\mathrm{MM}}\right)+E_{\mathrm{MM}}\left(\boldsymbol{r}_{\mathrm{MM}}\right) \\
= & E_{1}\left(\boldsymbol{r}_{\mathrm{QM}}, v_{\mathrm{MM}}^{0}\right)+\sum_{i \in \mathrm{QM}} Q_{i}^{0} v_{\mathrm{MM}}\left(\boldsymbol{r}_{\mathrm{QM}, i}\right)+\frac{1}{2} \sum_{i, j \in \mathrm{QM}}\left[v_{\mathrm{MM}}\left(\boldsymbol{r}_{\mathrm{QM}, i}\right)-v_{\mathrm{MM}}^{0}\left(\boldsymbol{r}_{\mathrm{QM}, i}\right)\right] \chi_{i j}\left[v_{\mathrm{MM}}\left(\boldsymbol{r}_{\mathrm{QM}, j}\right)-v_{\mathrm{MM}}^{0}\left(\boldsymbol{r}_{\mathrm{QM}, j}\right)\right] \\
& +E_{\mathrm{QM} / \mathrm{MM}, \mathrm{vdw}}\left(\boldsymbol{r}_{\mathrm{QM}}, \boldsymbol{r}_{\mathrm{MM}}\right)+E_{\mathrm{QM} / \mathrm{MM}, \mathrm{cov}}\left(\boldsymbol{r}_{\mathrm{QM}}, \boldsymbol{r}_{\mathrm{MM}}\right)+E_{\mathrm{MM}}\left(\boldsymbol{r}_{\mathrm{MM}}\right) .
\end{aligned}
$$

By taking the derivatives of each individual term, the QM gradient is

$$
\begin{aligned}
\frac{\partial \widetilde{E}\left(\boldsymbol{r}_{\mathrm{QM}}, \boldsymbol{r}_{\mathrm{MM}}\right)}{\partial \boldsymbol{r}_{\mathrm{QM}, i}}= & \frac{\partial E_{1}\left(\boldsymbol{r}_{\mathrm{QM}}, v_{\mathrm{MM}}^{0}\right)}{\partial \boldsymbol{r}_{\mathrm{QM}, i}}+Q_{Q M, i}^{0} \frac{\partial v_{\mathrm{MM}}\left(\boldsymbol{r}_{\mathrm{QM}, i}\right)}{\partial \boldsymbol{r}_{\mathrm{QM}, i}}+\sum_{j \in \mathrm{QM}}\left[v_{\mathrm{MM}}\left(\boldsymbol{r}_{\mathrm{QM}, j}\right)-v_{\mathrm{MM}}^{0}\left(\boldsymbol{r}_{\mathrm{QM}, j}\right)\right] \chi_{j i} \frac{\partial v_{\mathrm{MM}}\left(\boldsymbol{r}_{\mathrm{QM}, i}\right)}{\partial \boldsymbol{r}_{\mathrm{QM}, i}} \\
& +\frac{\partial\left(E_{\mathrm{QM} / \mathrm{MM}, \mathrm{vdw}}\left(\boldsymbol{r}_{\mathrm{QM}}, \boldsymbol{r}_{\mathrm{MM}}\right)+E_{\mathrm{QM} / \mathrm{MM}, \mathrm{cov}}\left(\boldsymbol{r}_{\mathrm{QM}}, \boldsymbol{r}_{\mathrm{MM}}\right)\right)}{\partial \boldsymbol{r}_{\mathrm{QM}, i}} .
\end{aligned}
$$


The derivative $\left[\partial E_{1}\left(\boldsymbol{r}_{\mathrm{QM}}, v_{\mathrm{MM}}^{0}\right)\right] / \partial \boldsymbol{r}_{\mathrm{QM}, i}$ is obtained from the $\mathrm{QM}$ analytical gradient and $\left[\partial v_{\mathrm{MM}}\left(\boldsymbol{r}_{\mathrm{QM}, i}\right)\right] / \partial \boldsymbol{r}_{\mathrm{QM}, i}$ is obtained directly from Eq. (26). As shown previously, this polarizable QM ESP charge model yields accurate QM/MM electrostatic energies even in drastically different $\mathrm{MM}$ environments. ${ }^{49}$ Again, we choose the mean electrostatic MM field from MD simulations of the MM subsystem to build the reference potential $v_{\mathrm{MM}}^{0}\left(\boldsymbol{r}_{\mathrm{QM}}\right)$.

\section{3. $Q M$ point charges with polarization due to $M M$ and $Q M$ atoms}

A further improvement to the $\mathrm{QM}$ polarization interaction can be made by including the polarization from QM atoms. That is, QM ESP charge $Q_{i}$ responds linearly to perturbations in both the MM environment and the QM geometry according to the equation

$$
\begin{aligned}
Q_{i}\left(\boldsymbol{r}_{\mathrm{QM}}, v_{\mathrm{MM}}\right)= & Q_{\mathrm{QM}, i}^{0}+\sum_{j \in \mathrm{QM}} \chi_{i j}\left[v_{\mathrm{MM}}\left(\boldsymbol{r}_{\mathrm{QM}, j}^{0}\right)\right. \\
& \left.-v_{\mathrm{MM}}^{0}\left(\boldsymbol{r}_{\mathrm{QM}, j}^{0}\right)\right]+\sum_{j \in \mathrm{QM}} \kappa_{i j}\left[\boldsymbol{r}_{\mathrm{QM}, j}-\boldsymbol{r}_{\mathrm{QM}, j}^{0}\right] .
\end{aligned}
$$

Here, a second kernel is introduced to account for the response of the QM ESP charges to QM geometric perturbations, ${ }^{25}$

$$
\kappa_{i j}=\left(\frac{\partial Q_{\mathrm{QM}, i}}{\partial \boldsymbol{r}_{\mathrm{QM}, j}}\right)_{N} .
$$

Because the QM atoms are frozen during the course of the MD simulations, this second response kernel does not appear in the QM/MM energy in Eq. (36). However, because this kernel correctly describes the change in the QM polarizations with respect to the geometric change, the QM gradient computed from Eq. (36) is now accurate [or exact for the energy of Eq. (36)] and contains an additional term from $\kappa_{i j}$. Compared to Eq. (37), the QM gradient is now

$$
\begin{aligned}
\frac{\partial \tilde{E}\left(\boldsymbol{r}_{\mathrm{QM}}, \boldsymbol{r}_{\mathrm{MM}}\right)}{\partial \boldsymbol{r}_{\mathrm{QM}, i}}= & \frac{\partial E_{1}\left(\boldsymbol{r}_{\mathrm{QM}}, v_{\mathrm{MM}}^{0}\right)}{\partial \boldsymbol{r}_{\mathrm{QM}, i}}+Q_{\mathrm{QM}, i}^{0} \frac{\partial v_{\mathrm{MM}}\left(\boldsymbol{r}_{\mathrm{QM}, i}\right)}{\partial \boldsymbol{r}_{\mathrm{QM}, i}}+\sum_{j \in \mathrm{QM}} \kappa_{i j}\left[v_{\mathrm{MM}}\left(\boldsymbol{r}_{\mathrm{QM}, j}\right)-v_{\mathrm{MM}}^{0}\left(\boldsymbol{r}_{\mathrm{QM}, j}\right)\right] \\
& +\sum_{j \in \mathrm{QM}}\left[v_{\mathrm{MM}}\left(\boldsymbol{r}_{\mathrm{QM}, j}\right)-v_{\mathrm{MM}}^{0}\left(\boldsymbol{r}_{\mathrm{QM}, j}\right)\right] \chi_{j i} \frac{\partial v_{\mathrm{MM}}\left(\boldsymbol{r}_{\mathrm{QM}, i}\right)}{\partial \boldsymbol{r}_{\mathrm{QM}, i}}+\frac{\partial\left(E_{\mathrm{QM} / \mathrm{MM}, \mathrm{vdw}}\left(\boldsymbol{r}_{\mathrm{QM}}, \boldsymbol{r}_{\mathrm{MM}}\right)+E_{\mathrm{QM} / \mathrm{MM}, \mathrm{cov}}\left(\boldsymbol{r}_{\mathrm{QM}}, \boldsymbol{r}_{\mathrm{MM}}\right)\right)}{\partial \boldsymbol{r}_{\mathrm{QM}, i}}
\end{aligned}
$$

Implementation of the polarizable QM ESP charge model described in Eqs. (32) and (38) improves the accuracy of the QM/MM energy and gradient. However, higher accuracy comes at an increased computational cost due to the calculation of the two response kernels. To compute the kernels, the QM ESP charges must first be analytically differentiable with respect to geometric perturbations. Then, one must solve coupled-perturbed Kohn-Sham equations. The first problem has been overcome by a recently developed analytical ESP fitting model, ${ }^{63}$ and the second problem has also been solved in our laboratory. ${ }^{64}$ The cost for solving the coupledperturbed Kohn-Sham equations is usually of the same order as a frequency calculation, which is expensive but still affordable for most QM systems of interest. To reduce the computational expense, one could begin with the simple nonpolarizable charge model to obtain paths of reasonable accuracy and later switch to more accurate calculations with polarizable QM ESP charges.

\section{E. Optimization of the reaction path on the QM PMF}

A reaction path provides a clear picture of a chemical reaction mechanism as well as quantitative information required for reaction rate calculations. It is thus important to implement an efficient method for determining reaction paths in association with the application of the QM/MM-MFEP method. For this work we consider optimization algorithms based on a chain-of-states, or discrete, representation of the path. Previously, the nudged elastic band (NEB) (Ref. 65) and the Ayala-Schlegel second-order minimum energy path (MEP) (Ref. 66) methods were used. ${ }^{49}$ Our experience indicates that the NEB method is simple to implement but converges slowly and has difficulty locating transition states. The MEP method can converge to the correct path, but its application to large molecular systems is troublesome because it requires an initial guess that is close to the exact path. As an alternative, a quadratic string method (QSM) (Ref. 67) was developed in our laboratory which has been shown to yield better performance than the NEB method. This method has been employed here as the main algorithm for path optimization.

For efficiency considerations, the whole path optimization process is usually separated into two stages. In the first stage, independent minimum free-energy minimizations are carried out for the system in the reactant and product valleys. In this process, the reference QM conformation used in Eq. (16) is taken from the optimized structure in the previous step, i.e., $\boldsymbol{r}_{\mathrm{QM}}^{\mathrm{ref}(n-1)}=\boldsymbol{r}_{\mathrm{QM}}^{(n-1)}$. The optimization of the QM geometry in a fixed MM ensemble, i.e., Eq. (15), is carried out 


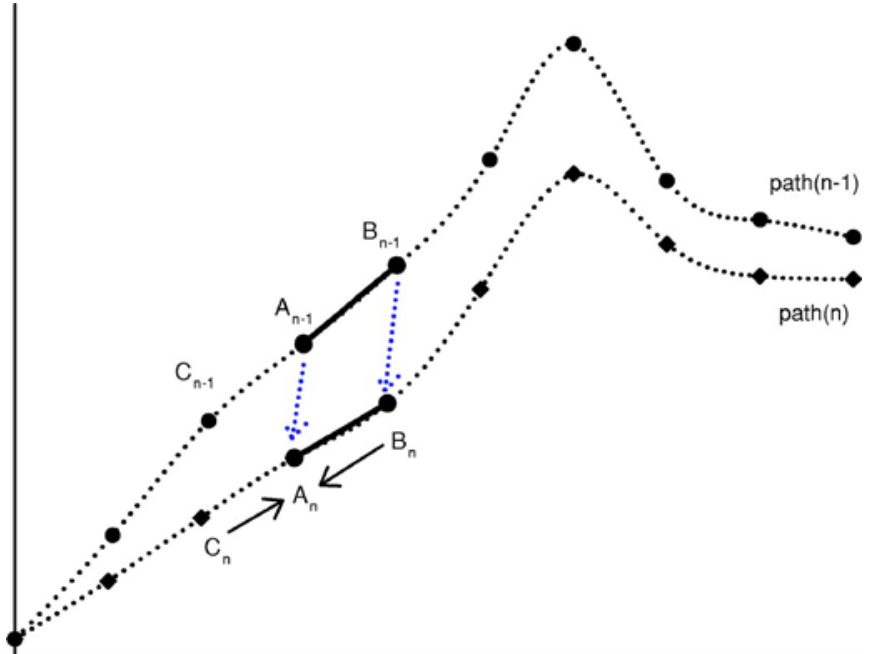

FIG. 1. (Color online) Path optimization scheme and different choices for the reference QM conformations in the PMF and PMF gradient calculations of the QM/MM-MFEP method.

with GAUSSIAN03 (Ref. 33) using the keyword "external." Accordingly, "loose" convergence criteria as defined in GAUSSIAN03 are used. From step (3) of Algorithm 2, convergence is achieved when the free-energy variation of a newly optimized $\mathrm{QM}$ conformation, $\boldsymbol{r}_{\mathrm{QM}}^{(n)}$, with respect to the previously optimized QM conformation, $\boldsymbol{r}_{\mathrm{QM}}^{(n-1)}$, is less than 1.0 $\mathrm{kcal} / \mathrm{mol}$.

In the second stage of the path optimization, QSM is employed to optimize the entire reaction path with the preoptimized structures of the reactant and product states used as fixed anchoring points. For all other conformations on the path, the PMF and PMF gradient are computed according to Eqs. (16) and (17). In these calculations, there are two possible choices for the reference QM conformations. As shown in Fig. 1, for a conformation state $\boldsymbol{A}_{n}$ on the $n$th path, its relative PMF could be computed with neighboring conformation states $\boldsymbol{B}_{\boldsymbol{n}}$ and $\boldsymbol{C}_{\boldsymbol{n}}$ acting as reference states, or with its optimized conformation from the previous path, $\boldsymbol{A}_{\boldsymbol{n}-1}$, acting as the reference state. Both will yield the same result. However, we choose to use neighboring conformational states as reference states so we do not have to store the trajectory of MM conformations from the previous optimization step in order to perform the required ensemble averaging described in Eq. (16).

As mentioned in the previous section, for efficiency considerations, one could begin the path optimization process with the simple QM ESP charge model and later increase the precision by using a polarizable QM ESP charge model. Further savings in computation time can be achieved by realizing that a high-precision path is not necessary to obtain the reaction energetics. Instead, if one can obtain accurate structures for the stationary points on the path, i.e., reactant, product, and transition state, an approximate path can be easily interpolated between adjacent stationary points. This path can then be minimized with QSM even without the need for complete convergence. This approximate QSM path is usually accurate enough to carry out free-energy perturbation calculations and obtain the activation free energy.

\section{F. Dynamic contributions from the QM subsystem}

In the QM/MM-MFEP method, the dynamic contributions of the QM subsystem have been left out of the QM $\mathrm{PMF}$ expression as the temperature of the QM subsystem is essentially $0 \mathrm{~K}$. The dynamic contributions are necessary for recovering the full free energy of the system. For stationary points on the reaction path, an effective approach is the harmonic approximation, which assumes that the PMF surface of the system is quadratic around the stationary points. This assumption allows the missing free-energy components to be included through a vibrational frequency analysis.

Specifically, one first computes $\left[\partial^{2} A\left(\boldsymbol{r}_{\mathrm{QM}}\right)\right] / \partial \boldsymbol{r}_{\mathrm{QM}, i} \partial \boldsymbol{r}_{\mathrm{QM}, j}$, the second derivatives of the PMF surface with respect to the QM geometries (the Hessian matrix). This matrix can be computed numerically or analytically as ${ }^{49}$

$$
\begin{aligned}
\frac{\partial^{2} A\left(\boldsymbol{r}_{\mathrm{QM}}\right)}{\partial \boldsymbol{r}_{\mathrm{QM}, i} \partial \boldsymbol{r}_{\mathrm{QM}, j}}= & \left\langle\frac{\partial^{2} E_{\mathrm{QM} / \mathrm{MM}}}{\partial \boldsymbol{r}_{\mathrm{QM}, i} \partial \boldsymbol{r}_{\mathrm{QM}, j}}\right\rangle_{E, \boldsymbol{r}_{\mathrm{MM}}} \\
& -\beta\left[\left\langle\frac{\partial E_{\mathrm{QM} / \mathrm{MM}}}{\partial \boldsymbol{r}_{\mathrm{QM}, i}} \frac{\partial E_{\mathrm{QM} / \mathrm{MM}}}{\partial \boldsymbol{r}_{\mathrm{QM}, j}}\right\rangle_{E, \boldsymbol{r}_{\mathrm{MM}}}\right. \\
& \left.-\left\langle\frac{\partial E_{\mathrm{QM} / \mathrm{MM}}}{\partial \boldsymbol{r}_{\mathrm{QM}, i}}\right\rangle_{E, \boldsymbol{r}_{\mathrm{MM}}}\left\langle\frac{\partial E_{\mathrm{QM} / \mathrm{MM}}}{\partial \boldsymbol{r}_{\mathrm{QM}, j}}\right\rangle_{E, \boldsymbol{r}_{\mathrm{MM}}}\right] .
\end{aligned}
$$

Diagonalization of the mass-weighted Hessian matrix yields $3 N$ eigenvalues $\left\{\lambda_{i}\right\}$ and the associated eigenvectors (modes). For a first-order saddle point such as the transition state of a reaction, there is one negative eigenvalue whose corresponding eigenvector points along the direction of the reaction coordinate. If the molecule is isolated, the eigenvalue of the six translational and rotational modes should approach zero in the calculation. For a QM subsystem in solution or embedded in an enzyme environment, the six translational and rotational degrees of freedom can no longer be separated from other motions because of the coupled interactions between the QM and MM subsystems. Therefore, these librational motions of the QM subsystem must also be included in the calculations as (low-frequency) vibrations.

The positive eigenvalues of the mass-weighted Hessian matrix can be converted into harmonic vibrational frequencies $\left\{v_{i}\right\}$ through the equation

$$
v_{i}=\sqrt{\lambda_{i} / 4 \pi^{2}} .
$$

By treating each harmonic mode as a quantum harmonic oscillator, one can compute the entropic contributions by ${ }^{68}$

$$
S_{\mathrm{vib}}=k \sum_{i}\left[\frac{\hbar v_{i} / k T}{\exp \left(\hbar v_{i} / k T\right)-1}-\ln \left(1-\exp \left(-\hbar v_{i} / k T\right)\right)\right]
$$

The harmonic motions also contribute to the average energy or enthalpy of the system as 


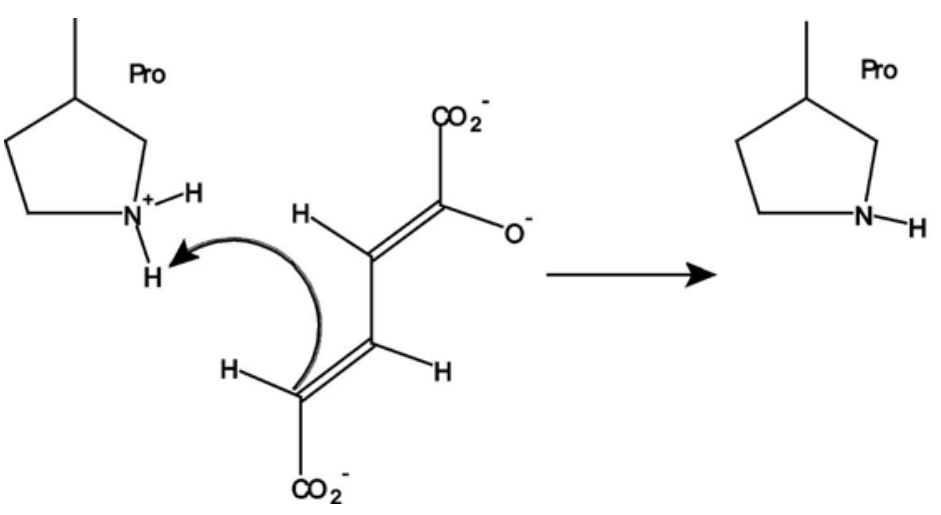

$E_{\mathrm{vib}}=k \sum_{i}\left[\frac{1}{2} \frac{\hbar v_{i}}{k}+\frac{\hbar v_{i} / k}{\exp \left(\hbar v_{i} / k T\right)-1}\right]$

The first term on the right-hand side is the zero-point energy of each mode, while the second term comes from the energy distribution of the harmonic motion.

The harmonic contributions described in Eqs. (43) and (44) are important to the QM/MM-MFEP simulation results. Under the harmonic assumption, the addition of these two terms to the QM/MM-MFEP free energies yields complete free energies for the stationary points of the reaction process. However, one cannot simply include the two terms and make direct comparisons to results from other theoretical methods such as umbrella sampling. For the conventional umbrella sampling approach, which treats all dynamics classically, the zero-point energy is not accounted for in the simulations, neither are the full $3 N$ degrees of freedom for the reactant and product. In umbrella sampling, the contributions of the restrained degrees of freedom are not included for all the conformations on the reaction path. While this is correct for the transition state, it misses one degree of freedom for the reactant, product, or any intermediate states. To enable a comparison between the QM/MM-MFEP and umbrella sampling results, the zero-point energy in Eq. (44) cannot be added to the QM/MM-MFEP result. The contribution of one or a few restrained degrees of freedom in umbrella sampling for the reactant and product states, nonetheless, cannot be analytically evaluated due to the difficulty of identifying the corresponding harmonic mode(s) in the QM/MM-MFEP results. To compute this term, simulation methods like the restrain-release approach ${ }^{69,70}$ could be applied to estimate the entropic cost for restraining specific degrees of freedom. The contribution of this single degree of freedom is expected to yield a small difference between the free energies computed with QM/MM-MFEP and umbrella sampling. Thus, it was not explored further in the current work.

One should also note that the harmonic approximation can only be applied to a single stationary state of the system. Doing so provides the entropic contribution from the vibrational motions within the specific conformational state. If the system possesses intramolecular rotations or multiple stable conformational states, an additional term called the configurational entropy should be included, ${ }^{71}$ as will be shown for the $\mathrm{S}_{\mathrm{N}} 2$ reaction.

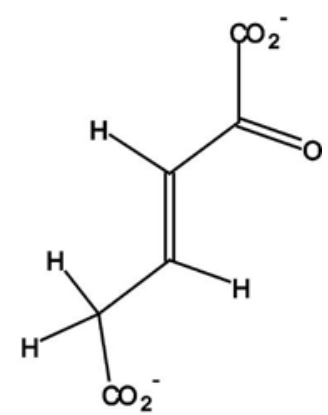

FIG. 2. Second proton transfer step of the reaction catalyzed by the enzyme 4-OT.

\section{SIMULATION DETAILS}

To illustrate the effectiveness of this iterative QM/MMMFEP method, we report here the simulation of two reaction processes: the $\mathrm{S}_{\mathrm{N}} 2$ reaction of $\mathrm{CH}_{3} \mathrm{Cl}+\mathrm{Cl}^{-}$as an example of an aqueous solution process, and the second proton transfer step catalyzed by the enzyme 4OT as an example of an enzymatic reaction. For the former reaction, we computed the activation free energy by two different methods to make the comparison: one involves direct QM/MM dynamics with umbrella sampling, and the other is the QM/MM-MFEP method developed in the current work. For the latter reaction, the results obtained from the QM/MM-MFEP simulation are compared with previous work.

All MD simulations were carried out with the program SIGMA, ${ }^{26,49,72,73}$ while all QM calculations were performed with GAUSSIAN03. ${ }^{33}$ The ESP fitting was carried out with our recently developed method, ${ }^{63}$ which makes the analytical calculation of the two response kernels feasible. In all the simulations where dual cutoffs were used, the charges of the $\mathrm{MM}$ atoms within the short-range cutoff were included in the QM calculations of the energies, gradients, and response kernels; hence, their polarization effects on the QM atoms were explicitly treated. Polarization effects from other MM atoms within the long-range cutoff were not considered. Their electrostatic interactions with the QM atoms were simply considered as Coulombic interactions between the MM point charges and the QM ESP charges.

For the $\mathrm{S}_{\mathrm{N}} 2$ process, the solute, i.e., the complex of $\mathrm{CH}_{3} \mathrm{Cl}$ and $\mathrm{Cl}^{-}$, was solvated in a cubic box with dimensions $48 \times 48 \times 48 \AA^{3}$ that contained 3600 tip3p water molecules. ${ }^{74}$ The CHARMM27 force field was used to describe the van der Waals interactions between the solute and the solvent molecules. ${ }^{75}$ For obvious reasons, one modification was made such that the atom type CLA was used for both chlorine and the chloride anion. The multiple-time-step $\operatorname{algorithm}^{76,77}$ was used for dynamic integration with a basic integration step size of 1 fs. A dual cutoff of 9 and $15 \AA$ was used for separating the interactions: the integration step size was 1 fs for short-range interactions, and 3 fs for mediumrange interactions. The nonbond pair lists were updated every 12 integration steps. The system was simulated under NVT conditions, with the temperature restrained at $300 \mathrm{~K}$ using a Berendsen thermostat. ${ }^{78}$ In the QM/MM-MFEP simulations, each period of MD sampling lasted for 90 ps in 


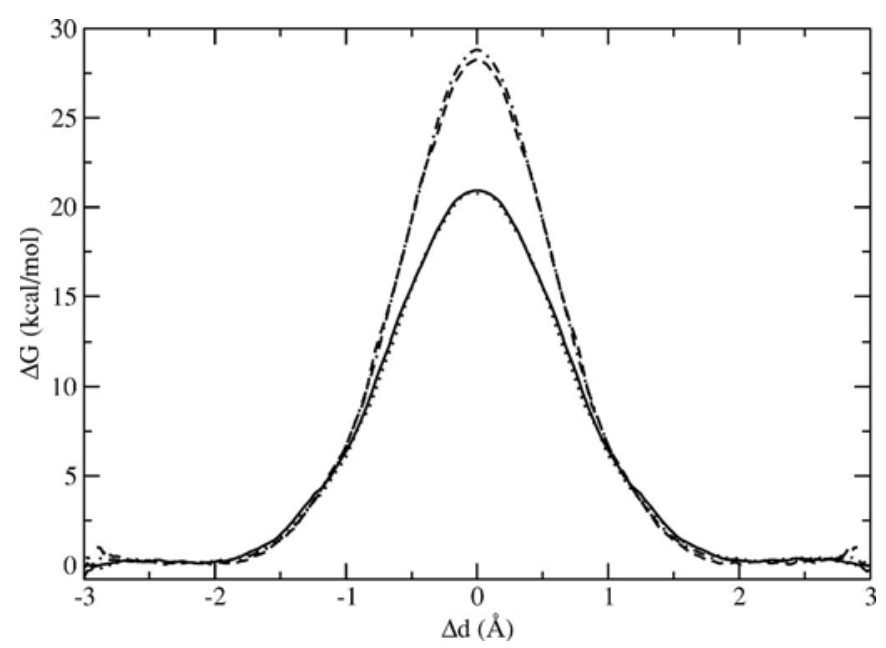

FIG. 3. Reaction free energies for $\mathrm{Cl}^{-}+\mathrm{CH}_{3} \mathrm{Cl}$ in solution simulated by umbrella sampling and WHAM analysis. The QM methods used were: MP2/6-31 + G* (dot-dash), MP2/6-311 + G* (dash), B3LYP/6-31+ G* (solid), and B3LYP/6-311+ $\mathrm{G}^{*}$ (dot).

the initial cycles, and $120 \mathrm{ps}$ in the final cycles for greater accuracy.

One purpose of the current study is to show that our QM/MM-MFEP method can generate reaction energetics of comparable accuracy to the direct classical sampling approach. Instead of comparing to an indirect experimental estimate of the reaction free-energy barrier, umbrella sampling was carried out to compute the barrier with direct QM/MM dynamics sampling for the complex of $\mathrm{CH}_{3} \mathrm{Cl}$ and $\mathrm{Cl}^{-}$. For such a small system, on-the-fly QM calculations at each MD step are affordable. The difference between the two $\mathrm{C}-\mathrm{Cl}$ bond lengths, $\Delta d=d_{\mathrm{C}-\mathrm{Cl}_{1}}-d_{\mathrm{C}_{-\mathrm{Cl}}}$, was used as the reaction order parameter. The distributions of the bond length differences were processed by the weighted histogram analysis method (WHAM). ${ }^{79,80}$ Two different QM methods, specifically B3LYP (Refs. 81 and 82 ) and MP2, ${ }^{83}$ were used to examine how the results vary with respect to the level of theory. Two common basis sets, $6-31+\mathrm{G}^{*}$ and $6-311+\mathrm{G}^{*}$, were used to examine the basis set dependence. A total of 25 sampling windows was used for the umbrella sampling. The centers of the harmonic potential were at $\Delta d=-2.5,-2.2$, $-1.9,-1.6,-1.4,-1.2,-1.0,-0.8,-0.6,-0.45,-0.3,-0.15$, $0.0,0.15,0.3,0.45,0.6,0.8,1.0,1.2,1.4,1.6,1.9,2.2$, and $2.5 \AA$. The force constants were $20,20,20,40,60,80,120$, $140,160,180,180,200,240,200,180,180,160,140,120$, $80,60,40,20,20$, and $20 \mathrm{kcal} /\left(\mathrm{mol}^{*} \AA^{2}\right)$. The results from umbrella sampling with B3LYP/6-31 $+\mathrm{G}^{*}$ were used as a reference for the QM/MM-MFEP simulations, in which the same QM method was used.

In the QM/MM-MFEP simulations of the $\mathrm{S}_{\mathrm{N}} 2$ process, two conformations were selected from the trajectories of the umbrella sampling simulations as the initial conformations of the reactant state and product state, respectively. Each conformation was optimized with the iterative QM/MM-MFEP method. In each cycle, the solute was optimized in the fixed ensemble of MM conformations until the gradient and energy converged to the thresholds set in GAUSIAN03 with rootmean-square gradients less than $0.36 \mathrm{kcal} / \mathrm{mol} / \AA$. The en-

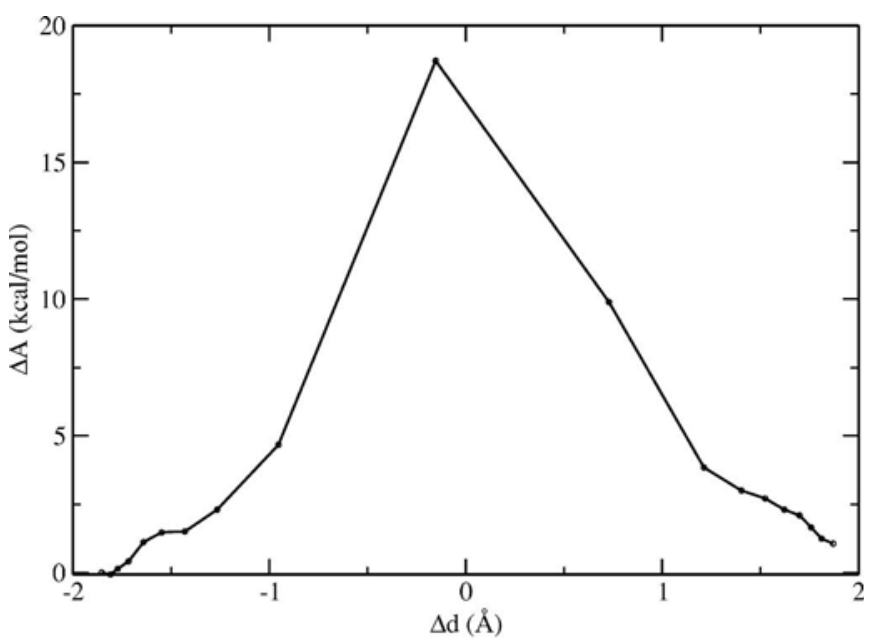

(a)

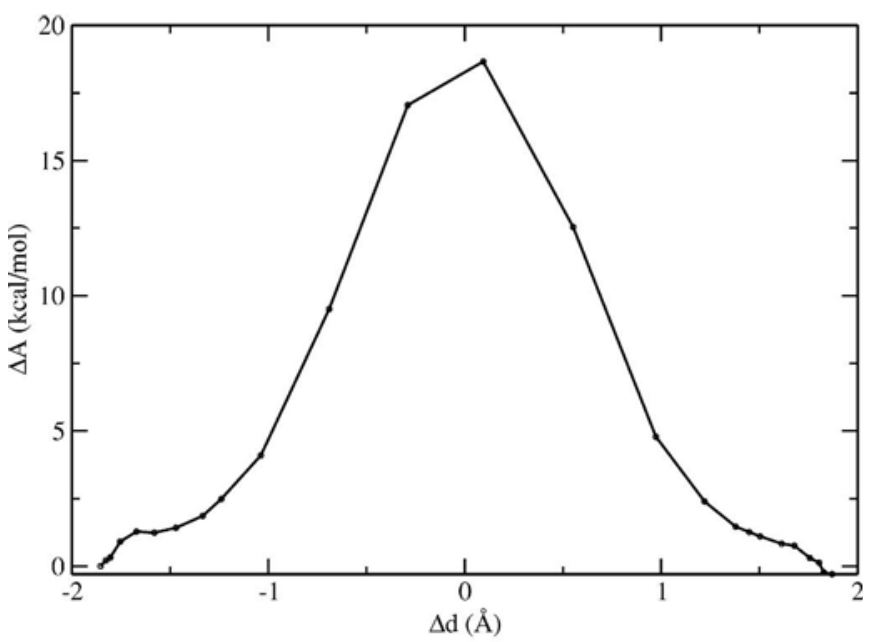

(b)

FIG. 4. Reaction free energies for $\mathrm{Cl}^{-}+\mathrm{CH}_{3} \mathrm{Cl}$ in solution simulated with QM/MM-MFEP and QSM. (a) Direct QSM optimization with the structures of the reactant and product state frozen; (b) two separate QSM simulations connecting the reactant state with the transition state, and the transition state with the product state.

tire optimization process was considered converged when the change in free energy between two sequential cycles was below $1.0 \mathrm{kcal} / \mathrm{mol}$. Usually this process required less than 10 cycles of QM/MM-MFEP minimizations.

After the conformations of the two end points were determined, conformations of 11 "intermediate" states were interpolated with GAUSSIAN03. These 11 conformations, plus the two end points, were used as the initial guess of the reaction path that was then optimized with QSM. For the reason discussed in Sec. II E, the QSM calculations were only carried out for three cycles. At that stage, the conformation with the highest free energy on the path was selected for transition state optimization on the free-energy surface. The QST3 algorithm implemented in GAUSSIAN03 was used to accomplish this task. ${ }^{66}$

With the structure of the transition state determined, two separate QSM calculations were carried out to determine the free-energy changes along the reaction path, one connecting the reactant state to the transition state, and the other connecting the transition state to the product state. Again, each 
TABLE I. Vibrational frequencies (in $\mathrm{cm}^{-1}$ ) of the transition state (TS) and reactant state (RS) of the complex $\mathrm{Cl}^{-}+\mathrm{CH}_{3} \mathrm{Cl}$ in solution.

\begin{tabular}{rrr}
\hline \hline Index & \multicolumn{1}{c}{ TS } & \multicolumn{1}{c}{ RS } \\
\hline 1 & -298.64 & 41.72 \\
2 & 66.37 & 49.44 \\
3 & 95.87 & 57.76 \\
4 & 106.46 & 66.09 \\
5 & 113.75 & 77.40 \\
6 & 125.46 & 109.97 \\
7 & 129.38 & 168.40 \\
8 & 214.60 & 175.90 \\
9 & 217.02 & 182.38 \\
10 & 234.07 & 708.32 \\
11 & 976.67 & 1039.97 \\
12 & 982.33 & 1052.31 \\
13 & 1125.74 & 1408.77 \\
14 & 1425.90 & 1497.86 \\
15 & 1429.94 & 1514.00 \\
16 & 3220.21 & 3146.16 \\
17 & 3411.26 & 3253.99 \\
18 & 3418.54 & 3263.34 \\
\hline \hline
\end{tabular}

simulation employed 11 middle conformations to build and optimize the path. Energies from the two half reactions were then pieced together to generate the full path.

The frequency analysis described in Sec. II F was carried out for the reactant and transition state of the $\mathrm{S}_{\mathrm{N}} 2$ reaction. The PMF Hessian matrix was computed by numerically differentiating the PMF gradient $\left[\partial A\left(\boldsymbol{r}_{\mathrm{QM}}\right)\right] / \partial \boldsymbol{r}_{\mathrm{QM}, i}$. The entropic contributions from vibrational motions were then computed and included into the free energy of the reaction process. The enthalpic contributions, without the zero-point energies in Eq. (44), were also computed for comparison with umbrella sampling.

The second proton transfer step in the reaction catalyzed

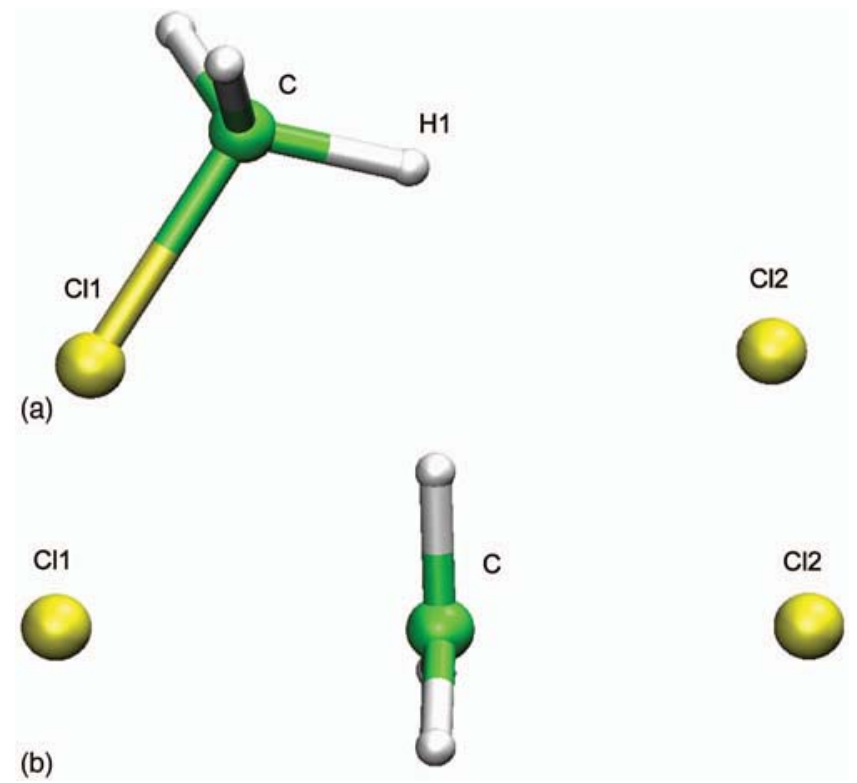

FIG. 5. (Color) Solution-phase stationary point structures of the $\mathrm{Cl}^{-}$ $+\mathrm{CH}_{3} \mathrm{Cl}$ complex optimized by the QM/MM-MFEP method: (a) reactant (and product) state; (b) transition state.
TABLE II. Geometric measurements of the reactant state (RS), product state (PS), and transition state (TS) for the reaction $\mathrm{Cl}^{-}+\mathrm{CH}_{3} \mathrm{Cl}$. Bond lengths are in $\AA$, and bond angles are in degrees.

\begin{tabular}{lccc}
\hline \hline Bond or angle & RS & PS & TS \\
\hline C-Cl1 & 1.81 & 3.68 & 2.30 \\
C-Cl2 & 3.66 & 1.81 & 2.32 \\
Cl2-C-Cl1 & 98.4 & 102.1 & 179.1 \\
\hline \hline
\end{tabular}

by 4OT was also simulated by the QM/MM-MFEP method (Fig. 2). Starting conformations for the reactant and product states were taken from previous work. ${ }^{23,27,40,42}$ The setup used in the current QM/MM-MFEP simulations was similar to the previous simulations. ${ }^{42}$ The system contained 5973 protein atoms described by the CHARMM 27 force field and 2408 tip3p water molecules. Harmonic restraints with a force constant of $40 \mathrm{kcal} /\left(\mathrm{mol}^{*} \AA^{2}\right)$ were applied to all heavy atoms that were further than $20 \AA$ from the $\mathrm{C} 3$ atom of the substrate. A dual cutoff of 8 and $40 \AA$ was used for all nonbonded interactions. Consistent with previous work, the QM system was described by the B3LYP/6-31G* level of theory and the QM/MM boundary was described using the pseudobond approach. ${ }^{39}$ The integration step size was $1 \mathrm{fs}$ for shortrange forces and $4 \mathrm{fs}$ for medium-range forces. The nonbond pair lists were updated every $16 \mathrm{MD}$ steps. The temperature of the system was held at $300 \mathrm{~K}$ with a Berendsen thermostat.

\section{RESULTS}

\section{A. $S_{\mathrm{N}} 2$ reaction}

The results from umbrella sampling with direct QM/MM dynamics sampling are shown in Fig. 3. Both the B3LYP and the MP2 calculations showed little dependence on the basis sets. The computed activation free energy was $20.7 \mathrm{kcal} / \mathrm{mol}$ with B3LYP/6-31+G*, and $28.7 \mathrm{kcal} / \mathrm{mol}$ with $\mathrm{MP} 2 / 6-31$ $+\mathrm{G} *$. The best estimate of the activation free energy for this process is $26.6 \mathrm{kcal} / \mathrm{mol},{ }^{84}$ but no direct experimental measurements have been reported. One must note that the ordinary umbrella sampling approach does not consider the contributions from the zero-point energy. Nonetheless, entropic contributions are implicitly included in the MD simulations. The result of $20.7 \mathrm{kcal} / \mathrm{mol}$ from umbrella sampling using the B3LYP/6-31+ $\mathrm{G}^{*}$ level of theory was used to compare against the iterative QM/MM-MFEP simulations.

The free energies from the QSM path optimization, both before and after the transition state optimization, are shown in Fig. 4. The converged free-energy difference between the reactant and the transition state is $18.7 \mathrm{kcal} / \mathrm{mol}$ without including the dynamic contributions from the QM subsystems. Inclusion of contributions from the harmonic vibrational motions computed via frequency calculations, (Table I) gives an activation free energy of $19.7 \mathrm{kcal} / \mathrm{mol}$ as the estimate from the QM/MM-MFEP method.

Umbrella sampling only simulates the classical dynamics of the QM/MM system. To make an effective comparison, we include contributions of the harmonic vibrational motions computed with frequency calculations, but without the contributions of the zero-point energy in Eq. (44). This 


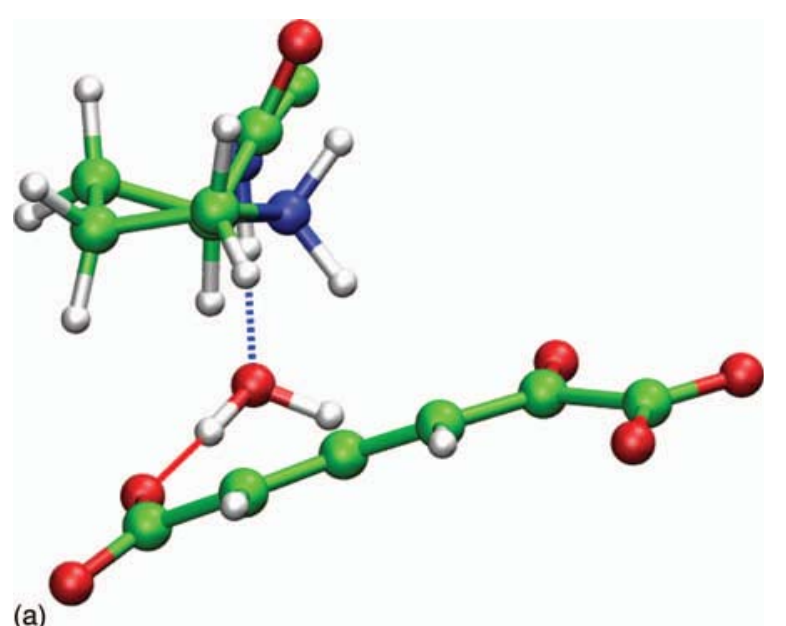

(a)

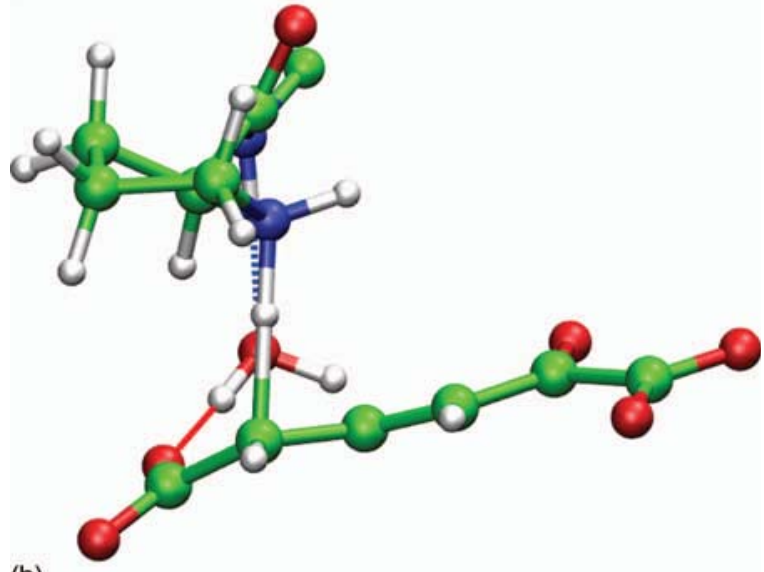

(b)

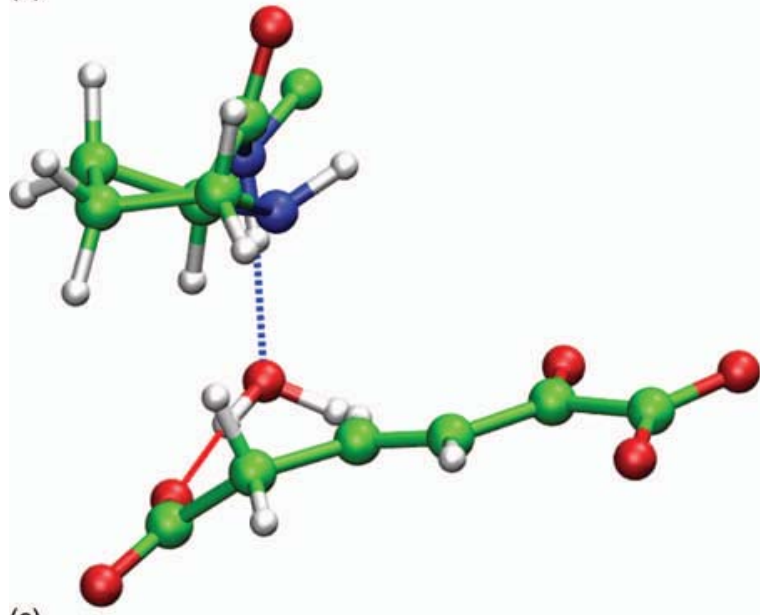

(c)

FIG. 6. (Color) Stationary point structures from the active site of 4OT optimized with the QM/MM-MFEP method: (a) reactant state; (b) transition state; and (c) product state.

yields an activation free energy of $20.4 \mathrm{kcal} / \mathrm{mol}$, in good agreement with the results from umbrella sampling (20.7 $\mathrm{kcal} / \mathrm{mol})$.

However, we need to consider two additional small contributions of opposite energetic effects: One originates from the rotational multiplicity of the reactant (and product) structure in the QM/MM-MFEP method. The $\mathrm{Cl}^{-}$ion has in fact equal possibilities of forming a weakly H-bonded complex with each of the three $\mathrm{C}-\mathrm{H}$ bonds [Fig. 5(a)]. In umbrella sampling, the three states can be adequately sampled in a

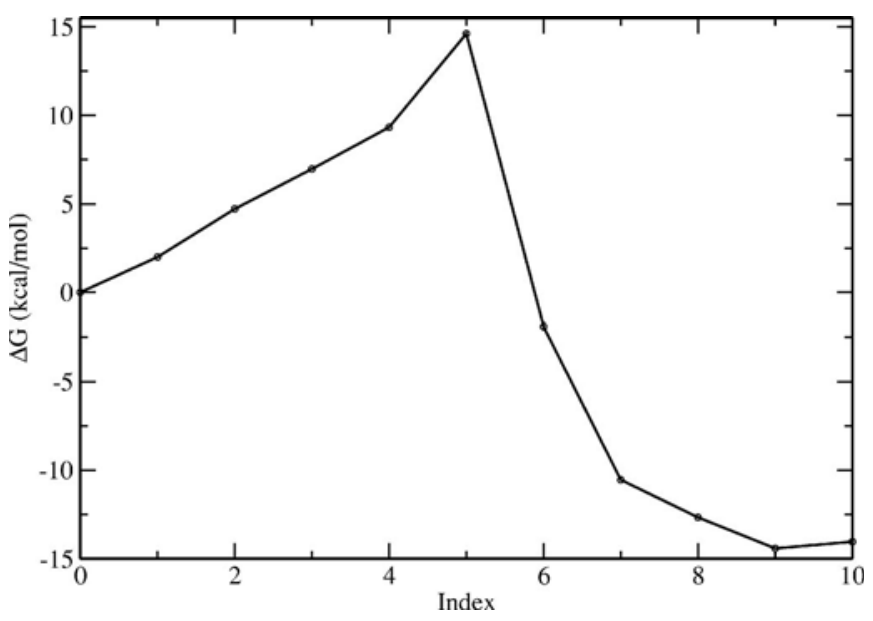

FIG. 7. Reaction free energies of the second proton transfer step of the reaction catalyzed by $4 \mathrm{OT}$ using QM/MM-MFEP and QSM.

simulation of $\sim 10$ ps. To account for this rotational multiplicity in QM/MM-MFEP, an additional entropic contribution of $k T \ln (1 / 3)=-0.65 \mathrm{kcal} / \mathrm{mol}$ should be added to the free energy of the reactant state of $20.4 \mathrm{kcal} / \mathrm{mol}$. This contribution yields an activation free energy of $21.1 \mathrm{kcal} / \mathrm{mol}$ for the QM/MM-MFEP method. The second contribution comes from the fact that the reactant state from umbrella sampling has one less configurational degree of freedom (the reaction coordinate). Often omitted in the literature, the contribution of the single, restrained degree of freedom in umbrella sampling for the reactant state cannot be precisely evaluated without additional sampling, ${ }^{69,70}$ but it will lower the free energy for the reactant state and raise the barrier of reaction for the umbrella sampling calculations.

The conformations of the reactant and transition state of the reaction of $\mathrm{CH}_{3} \mathrm{Cl}+\mathrm{Cl}^{-}$, optimized with the QM/MMMFEP method, are illustrated in Fig. 5 and the characteristic bonds and angles are listed in Table II. The slight difference between the structures of the reactant and product is due to fluctuations of the MM conformations. For the same reason, the transition state is slightly asymmetrical. One must note that, unless certain biased sampling methods were carried out on the MM subsystems, it would be extremely slow and computationally expensive to optimize to the putative symmetrical transition state. The slight deviation from the symmetrical structure, however, does not significantly affect the calculation of the free energies. As expected, the frequencies of the reactant state and transition state on the PMF surface reported in Table I clearly demonstrate a single negative frequency for the transition state.

\section{B. $40 T$ reaction}

The second proton transfer step of the reaction catalyzed by $4 \mathrm{OT}$ was also simulated with the new iterative QM/MMMFEP method. The optimized structures of the reactant, product, and transition state are shown in Fig. 6. The freeenergy change along the reaction path simulated using QSM is illustrated in Fig. 7. The QSM barrier is $14.7 \mathrm{kcal} / \mathrm{mol}$, in excellent agreement with the previous simulation result of $14.47 \mathrm{kcal} / \mathrm{mol}^{42}$ 

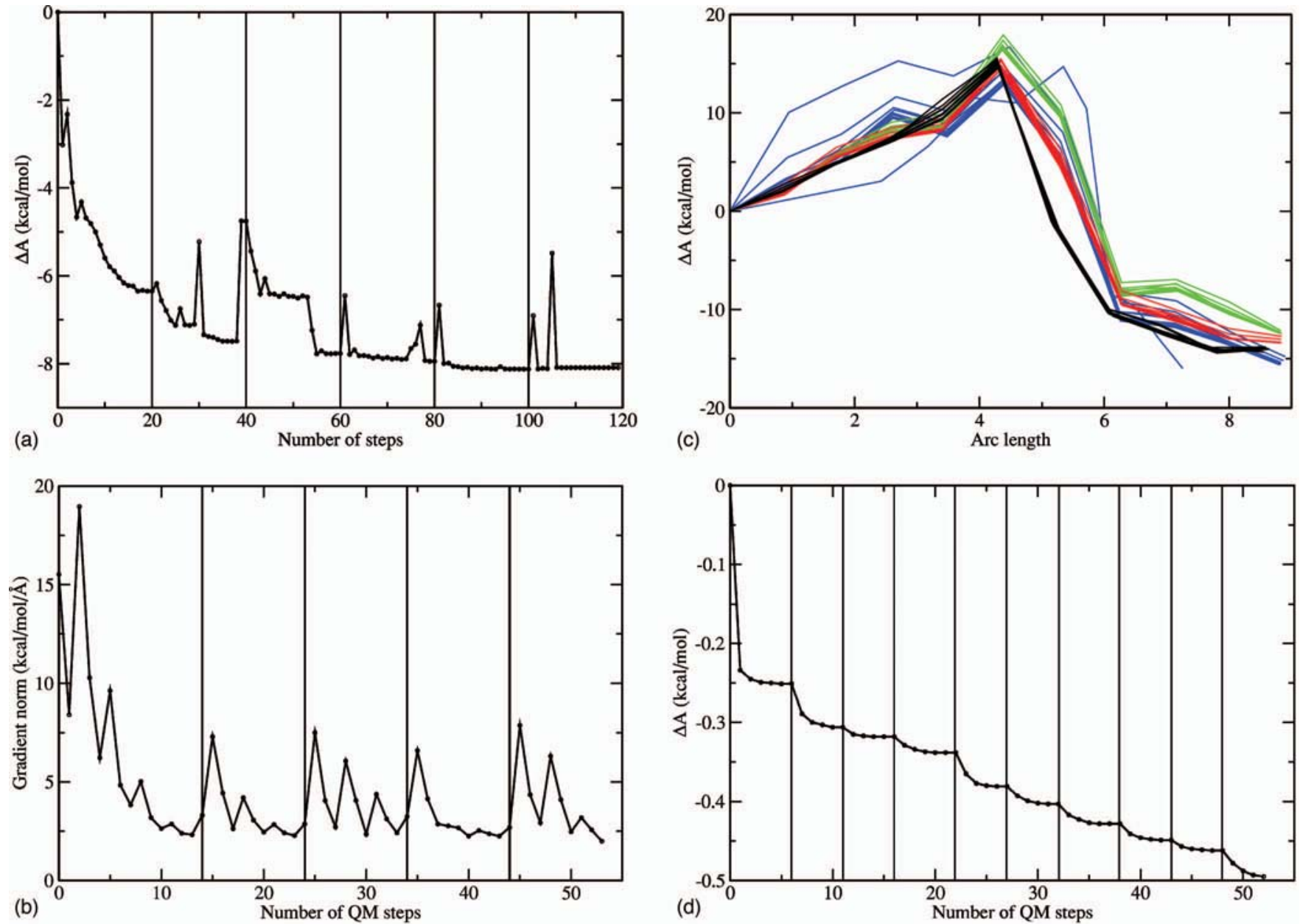

FIG. 8. (Color) Convergence of the QM/MM-MFEP and QSM optimizations for the $4 \mathrm{OT}$ and $\mathrm{S}_{\mathrm{N}} 2$ reactions. (a) Convergence of the relative free energies in the optimization of the reactant state of 4OT. Each vertical line indicates the start of a new cycle. The $X$-axis is the accumulated number of QM steps made by the QM optimization algorithm. Vertical lines indicate the onset of a new cycle of sequential MM sampling and QM optimization. The number of vertical lines is the number of times MM ensembles are generated. (b) Convergence of the norm of the gradient for one conformational state on the path of the 4OT reaction. (c) Evolution of the PMF profiles during successive cycles of QSM optimization of the 4OT reaction: cycle 1 (blue), cycle 2 (green), cycle 3 (red), cycle 4 (black). (d) Convergence of the relative free energies in the optimization of the reactant state for the $\mathrm{S}_{\mathrm{N}} 2$ reaction. Each vertical line indicates the start of a new cycle.

\section{DISCUSSION}

As expected, the current iterative QM/MM-MFEP method combined with the QSM path optimization algorithm significantly improves the convergence of reaction paths. To illustrate how the optimization converges in practical applications, we plot here the evolution of various characteristic quantities during the QM/MM-MFEP+QSM optimization of the proton transfer reaction catalyzed by the enzyme 4OT.

Figure 8(a) shows the variation of the (relative) free energy of the molecular system during the optimization of the reactant state. For each given ensemble of MM conformations, 20 QM steps of the QM optimization were carried out. A QM step is defined as a move of the QM subsystem made by the specific optimization algorithm, with the computational cost including one free energy/energy and gradient evaluation and the cost of the linear search. It is clear that after six cycles of sequential sampling and optimization using QM/MM-MFEP, the free energy of the system converges to a plateau. The spikes in the figure are the result of the optimization algorithm implemented in GAUSSIAN03, and are frequently observed in various molecular systems, especially for the first few steps of QM optimizations in the static MM ensembles. Figure 8(b) shows the variation of the norm of the gradient for an arbitrarily chosen conformation on the reaction path during the QM/MM-MFEP optimization process. The norm of the gradient converges at a similar rate to the free energy. In Fig. 8(c), we also show the evolution of the PMF profile for the whole reaction path in four cycles of QSM optimization. The conformations of the initial path were generated from simple linear interpolations between the optimized reactant and product state and were followed by simple energy minimizations. As a result, the free energies of those conformations fluctuate significantly throughout the reaction process. However, with QSM the PMF profile converges quickly.

For solution reactions, the convergence of the reaction path is even better than that for the enzymatic reaction. Figure 8(d) shows the changes of the relative free energy during the QM/MM-MFEP optimization of the reactant for the $S_{N} 2$ reaction. In each $\mathrm{MM}$ ensemble it usually takes less than ten optimization steps to attain full convergence in the QM optimization. One should note that, compared to Fig. 8(a), the 
magnitude of the change in the relative free energy is smaller in the $S_{N} 2$ reaction; however, the structural changes are nonnegligible. This result can also be partly understood from the flat PMF curve of the reactant and product valleys shown in Fig. 3. In general, the QM optimization in step (2b) of Algorithm 2 converges quickly in a few steps, even with the tight convergence criteria in GAUSSIAN03. This fast convergence can be understood because the short relaxation time and long-range isotropic distribution of the solvent molecules lead to the rapid convergence of the ensemble sampling of the MM environments. In contrast, because of the rugged energy landscape, the protein dynamics spans a broader range of timescales which requires longer MD simulations for enzymatic reactions.

Figures 8(a), 8(b), and 8(d) demonstrate the two advantages of our sequential sampling and optimization approach: First, the MM sampling only needed to be carried out at the end of each QM optimization within the finite ensemble. In contrast, the sampling is required at each step of a QM movement in the concurrent sampling and optimization method. Second, the total number of QM steps is greatly reduced because each QM optimization is carried out with the exact QM PMF and its gradients within the finite ensemble, allowing the classical optimization techniques to be most effective. On the other hand, in the concurrent MM sampling and QM optimization approach, the QM PMF and its gradients fluctuate during the QM steps, leading to slow convergence with classical optimization techniques.

As a prototype $\mathrm{S}_{\mathrm{N}} 2$ reaction, the self-exchange of $\mathrm{CH}_{3} \mathrm{Cl}+\mathrm{Cl}^{-}$has long been studied using various methods developed in different groups ${ }^{3,7,8,85-88}$ including the QM-FEP approach from the Jorgensen group, the EVB method from Warshel's group, and the MOVB method from Gao's group. The energetics of the reaction process, especially the solutesolvent interactions, have been analyzed in great detail and have been compared with the corresponding processes in enzymes in an attempt to answer questions regarding the origins of enzymatic proficiency. ${ }^{3,86}$ However, little is known about the structures of the reactant, product, and transition states. In fact, there have been few attempts to determine those structures, possibly because of the aforementioned difficulty in the theoretical treatment of the rapid fluctuations of the solvent molecules in solution. However, detailed knowledge concerning the specific structures of the stationary points and the reaction path would provide great benefit in the analysis of solvent-solute dynamics, such as nuclear tunneling effects in reaction processes.

With the inclusion of explicit solvent contributions, we determined here the structures of the stationary points. Surprisingly, the reactant adopted a bent, low-symmetry conformation, with the $\mathrm{Cl}^{-}$ion forming a weak hydrogen bond with one $\mathrm{C}-\mathrm{H}$ bond. On the contrary, if one carried out an optimization in the gas phase or with a continuum solvent model, the structure of the reactant state would eventually converge to a highly symmetric $\mathrm{C}_{3 v}$ conformation with the three heavy atoms $\mathrm{Cl}, \mathrm{C}$, and $\mathrm{Cl}$ collinear. ${ }^{88}$ Two observations further support this result that the low-symmetry structure has lower free energy. First is that in the umbrella sampling simulations we have carried out for determining the reaction barrier,

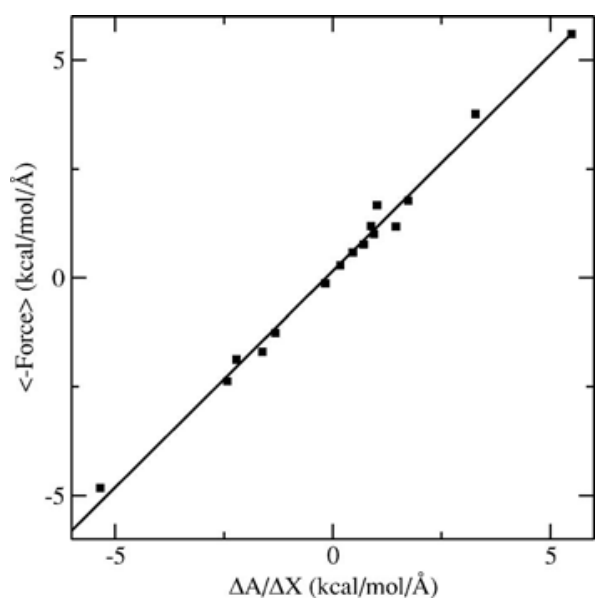

FIG. 9. Correlation between the free-energy gradient computed using analytical and numerical approaches. The $X$-axis contains the numerical freeenergy gradient computed for the $\mathrm{Cl}^{-}+\mathrm{CH}_{3} \mathrm{Cl}$ complex in solution, and the $Y$-axis contains the free-energy gradient computed with the analytical equations described in Sec. II, Theory.

$\mathrm{C}_{3 v}$-like conformations were barely observed. Second is that a set of short umbrella simulations has been performed which determined that the free energy of our low-symmetry structure is a few $(\sim 4) \mathrm{kcal} / \mathrm{mol}$ lower than the putative $\mathrm{C}_{3 v}$ structure. We believe that the stabilization of the lowsymmetry structure comes from the explicit solvent molecules in our simulations that provided a strong asymmetrical anisotropic stabilization for the reactant state. Such a structural difference for the reactant state strongly suggests the importance of including explicit solvent molecules in the simulation of solution reactions. It also highlights the risks of using conformations optimized in the gas phase or with a continuum solvent model for the study of solution or enzyme reactions.

Compared with the original QM/MM-MFEP method, the new method we have developed here contains two critical improvements: the use of a finite and fixed MM ensemble speeds up the overall convergence of the path optimization process; and the use of the polarizable ESP charge model improves the accuracy of the free-energy perturbation calculations and the PMF gradient. To illustrate the latter point, we compared the free-energy gradient computed using two different approaches (Fig. 9): analytical calculation as previously derived in Eq. (17), and numerical differentiation. As demonstrated for the $\mathrm{Cl}^{-}+\mathrm{CH}_{3} \mathrm{Cl}$ system, the results from the two approaches agree quite well, and thus ensure the success of the geometry optimization in the QM/MM-MFEP method.

The focus of the current study is to enhance the applicability of the QM/MM-MFEP method in the simulation of both enzyme and solution reactions. Of the many differences between reactions in solution and in enzymes, the predominant one from the perspective of simulation originates from the different dynamic properties of the reaction environment. In solution, the solvent molecules exchange positions rapidly around the reacting moieties, while the active site in an enzyme is more or less protected. Even though the active site is often regarded as quasistatic in the course of a reaction process, its conformation is also affected by the modulation of 
large-scale conformational changes of the enzyme molecule. Therefore, to model the reactions in solution or in enzymes, simulation methods should be able to yield converged contributions from the constantly fluctuating solvent molecules for reactions in solution, while at the same time allowing broad sampling of the enzyme conformational space. Both requirements are fully achieved in the QM/MM-MFEP method through the MD simulations and potential of mean force calculations.

One motivation for the development of the ab initio QM/ MM-MFEP methods was to simulate not only enzymatic but also solution reactions. This stems from the repeatedly observed fact that the catalytic proficiency of an enzyme is often determined by the degree of difficulty of the corresponding uncatalyzed reaction in solution. ${ }^{89,90}$ In addition to this observation, it has also been suggested that the catalytic power of an enzyme is mostly attributed to the preorganized electrostatic interactions in the structure of enzyme molecules, ${ }^{86,91,92}$ as well as conformational dynamics. ${ }^{18}$ Therefore, simulating how a reaction proceeds in solution can provide vital insight into the ultimate question of how an enzyme accomplishes a chemical process in a fraction of a second that would otherwise take millions of years without a catalyst. Despite the importance of the reference solution reactions, the number of solution reactions that have been the subject of theoretical scrutiny based on ab initio QM is far less than the number of enzymatic reactions. One reason for such an imbalance might be the fact that the methodology development for simulating solution reactions is not comparable with that for simulating enzyme reactions. Approaches that simplify the solvent contributions by a continuum solvent model can provide only very limited, and sometimes even incorrect, information concerning the reaction process. To correctly model the solvent contributions, one must include the solvent molecules explicitly in simulations.

\section{CONCLUSIONS}

In this report, we have presented a greatly enhanced $a b$ initio QM/MM-MFEP method for simulating accurate paths and energetics for reactions both in solution and in enzymes. Two developments have been made to improve the performance of the QM/MM-MFEP method, namely an iterative sequential MM sampling and QM optimization scheme in a finite and fixed-size ensemble of MM conformations to improve the optimization efficiency, and a fully polarizable QM ESP charge model to improve the accuracy of the QM energy and gradient calculations. The application of this method indicates that it provides accurate results in agreement with experimental data and other established simulation approaches. As costly ab initio QM methods become increasingly affordable for providing accurate information regarding the explicit structure and energetics of a reaction process, this method will play an important role in the simulation of reaction processes in solution and in enzymes.

In addition, our sequential sampling and optimization approach for MFEP optimization, constructed here using a finite and fixed ensemble in the calculation of free-energy difference, gradients, and optimization, may be directly ap- plied to any MFEP simulations based on other energy surfaces. As we have shown here, this will eliminate the problems in the conventional direct MFEP simulations where the convergence of the free-energy gradient is slow and intrinsic fluctuation of the sampling slows down the optimization.

\section{ACKNOWLEDGMENTS}

We gratefully acknowledge the National Institutes of Health for financial support.

${ }^{1}$ A. Warshel, Computer Modeling of Chemical Reactions in Enzymes and Solutions (Wiley \& Sons, New York, 1991).

${ }^{2}$ A. Warshel and M. Levitt, J. Mol. Biol. 103, 227 (1976).

${ }^{3}$ J.-K. Hwang, G. King, S. Creighton, and A. Warshel, J. Am. Chem. Soc. 110, 5297 (1988).

${ }^{4}$ M. Strajbl, G. Hong, and A. Warshel, J. Phys. Chem. B 106, 13333 (2002).

${ }^{5}$ A. Warshel, Annu. Rev. Biophys. Biomol. Struct. 32, 425 (2003).

${ }^{6}$ A. Warshel, P. K. Sharma, M. Kato, Y. Xiang, H. Liu, and M. H. M. Olsson, Chem. Rev. 106, 3210 (2006).

${ }^{7}$ J. Chandrasekhar, S. F. Smith, and W. L. Jorgensen, J. Am. Chem. Soc. 107, 154 (1985).

${ }^{8}$ W. L. Jorgensen, Acc. Chem. Res. 22, 184 (1989).

${ }^{9}$ O. Acevedo and W. L. Jorgensen, J. Am. Chem. Soc. 128, 6141 (2006).

${ }^{10}$ M. J. Field, P. A. Bash, and M. Karplus, J. Comput. Chem. 11, 700 (1990).

${ }^{11}$ P. A. Bash, M. J. Field, R. C. Davenport, G. A. Petsko, D. Ringe, and M. Karplus, Biochemistry 30, 5826 (1991).

${ }^{12}$ Q. Cui and M. Karplus, J. Chem. Phys. 112, 1133 (2000).

${ }^{13}$ Q. Cui and M. Karplus, J. Phys. Chem. B 104, 3721 (2000).

${ }^{14}$ Q. Cui and M. Karplus, J. Am. Chem. Soc. 123, 2284 (2001).

${ }^{15}$ M. Garcia-Viloca, J. Gao, M. Karplus, and D. G. Truhlar, Science 303, 186 (2004).

${ }^{16}$ J. Gao and X. Xia, Science 258, 631 (1992).

${ }^{17}$ J. Gao and D. G. Truhlar, Annu. Rev. Phys. Chem. 53, 467 (2002).

${ }^{18}$ J. L. Gao, Curr. Opin. Struct. Biol. 13, 184 (2003).

${ }^{19}$ J. Gao, S. Ma, D. T. Major, K. Nam, J. Pu, and D. G. Truhlar, Chem. Rev. 106, 3188 (2006)

${ }^{20}$ H. Y. Liu, Y. K. Zhang, and W. T. Yang, J. Am. Chem. Soc. 122, 6560 (2000).

${ }^{21}$ Y. Zhang, H. Liu, and W. Yang, J. Chem. Phys. 112, 3483 (2000).

${ }^{22}$ H. Liu, M. Elstner, E. Kaxiras, T. Frauenheim, J. Hermans, and W. Yang, Proteins Struct. Funct. Genet. 44, 484 (2001).

${ }^{23}$ G. A. Cisneros, H. Y. Liu, Y. K. Zhang, and W. T. Yang, J. Am. Chem. Soc. 125, 10384 (2003).

${ }^{24}$ H. Liu, Z. Lu, G. A. Cisneros, and W. Yang, J. Chem. Phys. 121, 697 (2004).

${ }^{25}$ Z. Lu and W. Yang, J. Chem. Phys. 121, 89 (2004).

${ }^{26} \mathrm{H}$. Hu and W. Yang, J. Chem. Phys. 123, 041102 (2005).

${ }^{27}$ G. A. Cisneros, M. Wang, P. Silinski, M. C. Fitzgerald, and W. T. Yang, J. Phys. Chem. A 110, 700 (2006).

${ }^{28}$ P. Hu and Y. K. Zhang, J. Am. Chem. Soc. 128, 1272 (2006).

${ }^{29}$ L. H. Wang, X. Y. Yu, P. Hu, S. Broyde, and Y. K. Zhang, J. Am. Chem. Soc. 129, 4731 (2007).

${ }^{30}$ G. M. Torrie and J. P. Valleau, J. Comput. Phys. 23, 187 (1977).

${ }^{31}$ B. Kuhn and P. A. Kollman, J. Am. Chem. Soc. 122, 2586 (2000).

${ }^{32}$ U. C. Singh and P. A. Kollmann, J. Comput. Chem. 7, 718 (1986).

${ }^{33}$ M. J. Frisch, G. W. Trucks, H. B. Schlegel et al.., Gaussian 03, Gaussian, Inc., Wallingford, CT, 2004.

${ }^{34}$ A. Shurki and A. Warshel, Adv. Protein Chem. 66, 249 (2003).

${ }^{35}$ T. Wesolowski and A. Warshel, J. Phys. Chem. 98, 5183 (1994).

${ }^{36}$ T. Wesolowski, R. P. Muller, and A. Warshel, J. Phys. Chem. 100, 15444 (1996).

${ }^{37}$ R. P. Muller and A. Warshel, J. Phys. Chem. 99, 17516 (1995).

${ }^{38}$ J. Bentzien, R. P. Muller, J. Florián, and A. Warshel, J. Phys. Chem. B 102, 2293 (1998).

${ }^{39}$ Y. Zhang, T.-S. Lee, and W. Yang, J. Chem. Phys. 110, 46 (1999).

${ }^{40}$ G. A. Cisneros, H. Y. Liu, Z. Y. Lu, and W. T. Yang, J. Chem. Phys. 122, 114502 (2005).

${ }^{41}$ M. Wang, Z. Lu, and W. Yang, J. Chem. Phys. 121, 101 (2004).

${ }^{42}$ G. A. Cisneros, M. Wang, P. Silinski, M. C. Fitzgerald, and W. Yang, 
Biochemistry 43, 6885 (2004)

${ }^{43}$ M. Wang, Z. Lu, and W. Yang, J. Chem. Phys. 124, 124516 (2006).

${ }^{44}$ S. L. Wang, P. Hu, and Y. K. Zhang, J. Phys. Chem. B 111, 3758 (2007).

${ }^{45}$ C. Corminboeuf, P. Hu, M. E. Tuckerman, and Y. K. Zhang, J. Am. Chem. Soc. 128, 4530 (2006).

${ }^{46}$ J. Kästner, H. M. Senn, S. Thiel, N. Otte, and W. Thiel, J. Chem. Theory Comput. 2, 452 (2006)

${ }^{47}$ M. Klahn, S. Braun-Sand, E. Rosta, and A. Warshel, J. Phys. Chem. B 109, 15645 (2005)

${ }^{48}$ G. A. Cisneros and W. T. Yang (unpublished).

${ }^{49}$ H. Hu, Z. Y. Lu, and W. T. Yang, J. Chem. Theory Comput. 3, 390 (2007).

${ }^{50}$ M. Nagaoka, N. Okuyama-Yoshida, and T. Yamabe, J. Phys. Chem. A 102, 8202 (1998)

${ }^{51}$ N. Okuyama-Yoshida, M. Nagaoka, and T. Yamabe, Int. J. Quantum Chem. 70, 95 (1998).

${ }^{52}$ N. Okuyama-Yoshida, K. Kataoka, M. Nagaoka, and T. Yamabe, J. Chem. Phys. 113, 3519 (2000).

${ }^{53}$ H. Hirao, Y. Nagae, and M. Nagaoka, Chem. Phys. Lett. 348, 350 (2001).

${ }^{54}$ P. Fleurat-Lessard and T. Ziegler, J. Chem. Phys. 123, 084101 (2005).

${ }^{55}$ S.-Y. Yang, I. Hristov, P. Fleurat-Lessard, and T. Ziegler, J. Phys. Chem. A 109, 197 (2005).

${ }^{56} \mathrm{G}$. Li and Q. Cui, J. Mol. Graph. Model. 24, 82 (2005).

${ }^{57}$ L. Maragliano, A. Fischer, E. Vanden-Eijnden, and G. Ciccotti, J. Chem. Phys. 125, 024106 (2006).

${ }^{58}$ M. Higashi, S. Hayashi, and S. Kato, J. Chem. Phys. 126, 144503 (2007).

${ }^{59}$ R. E. Miller, Optimization: Foundations and Applications (Wiley \& Sons, New York, 2000).

${ }^{60}$ T. H. Rod and U. Ryde, J. Chem. Theory Comput. 1, 1240 (2005).

${ }^{61}$ A. Morita and S. Kato, J. Am. Chem. Soc. 119, 4021 (1997).

${ }^{62}$ R. G. Parr and W. Yang, Density-Functional Theory of Atoms and Molecules (Oxford University Press, Oxford, 1994).

${ }^{63} \mathrm{H}$. Hu, Z. Lu, and W. Yang, J. Chem. Theory Comput. 3, 1004 (2007).

${ }^{64} \mathrm{Z}$. Lu, H. Hu, and W. Yang (to be published).

${ }^{65}$ H. Jónsson, G. Mills, and K. W. Jacobsen, Nudged Elastic Band Method for Finding Minimum Energy Paths of Transitions, Classical and Quantum Dynamics in Condensed Phase Simulations, edited by B. J. Berne, G. Ciccotti, D. F. Coker (World Scientific, Singapore, 1998).

${ }^{66}$ P. Y. Ayala and H. B. Schlegel, J. Chem. Phys. 107, 375 (1997).

${ }^{67}$ S. K. Burger and W. Yang, J. Chem. Phys. 124, 054109 (2006).

${ }^{68}$ T. L. Hill, An Introduction to Statistical Thermodynamics (Dover, New York, 1987).
${ }^{69}$ J. Hermans and L. Wang, J. Am. Chem. Soc. 119, 2707 (1997).

${ }^{70}$ M. Strajbl, Y. Y. Sham, J. Villa, Z. T. Chu, and A. Warshel, J. Phys. Chem. B 104, 4578 (2000).

${ }^{71}$ C. L. Brooks III, M. Karplus, and B. M. Pettitt, Proteins: A Theoretical Perspective of Dynamics, Structure, and Thermodynamics (Wiley \& Sons, New York, 1988).

${ }^{72} \mathrm{H}$. Hu, M. Elstner, and J. Hermans, Proteins Struct. Funct. Genet. 50, 451 (2003).

${ }^{73}$ G. Mann, R. H. Yun, L. Nyland, J. Prins, J. Board, and J. Hermans, in Computational Methods for Macromolecules: Challenges and Applications-Proceedings of the 3rd International Workshop on Algorithms for Macromolecular Modelling, edited by T. Schlick and H. H. Gan (Springer, Berlin, 2002), p. 129.

${ }^{74}$ W. L. Jorgensen, J. Chandrasekhar, J. D. Madura, R. W. Impey, and M. L. Klein, J. Chem. Phys. 79, 926 (1983).

${ }^{75}$ A. D. MacKerell, B. Brooks, C. L. Brooks, L. Nilsson, B. Roux, Y. Won, and M. Karplus, in The Encyclopedia of Computational Chemistry, edited by P. v. R. Schleyer et al.. (Wiley \& Sons, Chichester, 1998), Vol. 1, p. 271.

${ }^{76}$ M. E. Tuckerman, B. J. Berne, and G. J. Martyna, J. Chem. Phys. 97, 1990 (1992)

${ }^{77}$ T. Schlick, R. D. Skeel, A. T. Brünger, L. V. Kalé, J. A. Board, J. Hermans, and K. Schulten, J. Comput. Phys. 151, 9 (1999).

${ }^{78}$ H. J. C. Berendsen, J. P. M. Postma, W. F. van Gunsteren, A. DiNola, and J. R. Haak, J. Chem. Phys. 81, 3684 (1984).

${ }^{79}$ A. M. Ferrenberg and R. H. Swendsen, Phys. Rev. Lett. 63, 1195 (1989).

${ }^{80}$ S. Kumar, D. Bouzida, R. H. Swendsen, P. A. Kollman, and J. M. Rosenberg, J. Comput. Chem. 13, 1011 (1992).

${ }^{81}$ A. D. Becke, J. Chem. Phys. 98, 5648 (1993).

${ }^{82}$ C. Lee, W. Yang, and R. G. Parr, Phys. Rev. B 37, 785 (1988).

${ }^{83}$ M. Head-Gordon, J. A. Pople, and M. J. Frisch, Chem. Phys. Lett. 153, 503 (1988).

${ }^{84}$ D. J. McLennan, Aust. J. Chem. 31, 1897 (1978).

${ }^{85}$ P. A. Bash, M. J. Field, and M. Karplus, J. Am. Chem. Soc. 109, 8092 (1987).

${ }^{86}$ M. H. M. Olsson and A. Warshel, J. Am. Chem. Soc. 126, 15167 (2004).

${ }^{87}$ Y. R. Mo and J. L. Gao, J. Comput. Chem. 21, 1458 (2000).

${ }^{88}$ K. Ohmiya and S. Kato, Chem. Phys. Lett. 348, 75 (2001).

${ }^{89}$ A. Radzicka and R. Wolfenden, Science 267, 90 (1995).

${ }^{90}$ R. Wolfenden and M. J. Snider, Acc. Chem. Res. 34, 938 (2001).

${ }^{91}$ A. Warshel, J. Biol. Chem. 273, 27035 (1998).

${ }^{92}$ T. C. Bruice and P. Y. Bruice, J. Am. Chem. Soc. 127, 12478 (2005). 